\title{
Metagenomic Insights into the Uncultured Diversity and Physiology of Microbes in Four Hypersaline Soda Lake Brines
}

\author{
Charlotte D. Vavourakis ${ }^{1}$, Rohit Ghai ${ }^{2,3}$, Francisco Rodriguez-Valera ${ }^{2}$, \\ Dimitry Y. Sorokin ${ }^{4,5}$, Susannah G. Tringe ${ }^{6}$, Philip Hugenholtz ${ }^{7}$ and Gerard Muyzer ${ }^{1 *}$ \\ ${ }^{1}$ Microbial Systems Ecology, Department of Aquatic Microbiology, Institute for Biodiversity and Ecosystem Dynamics, \\ University of Amsterdam, Amsterdam, Netherlands, ${ }^{2}$ Evolutionary Genomics Group, Departamento de Producción Vegetal y \\ Microbiología, Universidad Miguel Hernández, San Juan de Alicante, Spain, ${ }^{3}$ Department of Aquatic Microbial Ecology, \\ Biology Centre of the Czech Academy of Sciences, Institute of Hydrobiology, České Budějovice, Czech Republic, ${ }^{4}$ Research \\ Centre of Biotechnology, Winogradsky Institute of Microbiology, Russian Academy of Sciences, Moscow, Russia, \\ ${ }^{5}$ Department of Biotechnology, Delft University of Technology, Delft, Netherlands, ${ }^{6}$ The Department of Energy Joint Genome \\ Institute, Walnut Creek, CA, USA, ${ }^{7}$ Australian Centre for Ecogenomics, School of Chemistry and Molecular Biosciences and \\ Institute for Molecular Bioscience, The University of Queensland, Brisbane, QLD, Australia
}

OPEN ACCESS

Edited by:

Andreas Teske,

University of North Carolina at Chapel Hill, USA

Reviewed by: Jinjun Kan,

Stroud Water Research Center, USA Xi-Ying Zhang,

Shandong University, China

${ }^{*}$ Correspondence: Gerard Muyzer g.muiizer@uva.n

Specialty section:

This article was submitted to

Extreme Microbiology,

a section of the journal

Frontiers in Microbiology

Received: 02 December 2015 Accepted: 08 February 2016 Published: 25 February 2016

Citation:

Vavourakis $C D$, Ghai $R$, Rodriguez-Valera F, Sorokin DY,

Tringe SG, Hugenholtz $P$ and

Muyzer G (2016) Metagenomic Insights into the Uncultured Diversity and Physiology of Microbes in Four

Hypersaline Soda Lake Brines.

Front. Microbiol. 7:211. doi: 10.3389/fmicb.2016.00211
Soda lakes are salt lakes with a naturally alkaline $\mathrm{pH}$ due to evaporative concentration of sodium carbonates in the absence of major divalent cations. Hypersaline soda brines harbor microbial communities with a high species- and strain-level archaeal diversity and a large proportion of still uncultured poly-extremophiles compared to neutral brines of similar salinities. We present the first "metagenomic snapshots" of microbial communities thriving in the brines of four shallow soda lakes from the Kulunda Steppe (Altai, Russia) covering a salinity range from 170 to $400 \mathrm{~g} / \mathrm{L}$. Both amplicon sequencing of $16 \mathrm{~S}$ rRNA fragments and direct metagenomic sequencing showed that the top-level taxa abundance was linked to the ambient salinity: Bacteroidetes, Alpha-, and Gamma-proteobacteria were dominant below a salinity of $250 \mathrm{~g} / \mathrm{L}$, Euryarchaeota at higher salinities. Within these taxa, amplicon sequences related to Halorubrum, Natrinema, Gracilimonas, purple non-sulfur bacteria (Rhizobiales, Rhodobacter, and Rhodobaca) and chemolithotrophic sulfur oxidizers (Thioalkalivibrio) were highly abundant. Twenty-four draft population genomes from novel members and ecotypes within the Nanohaloarchaea, Halobacteria, and Bacteroidetes were reconstructed to explore their metabolic features, environmental abundance and strategies for osmotic adaptation. The Halobacteria- and Bacteroidetes-related draft genomes belong to putative aerobic heterotrophs, likely with the capacity to ferment sugars in the absence of oxygen. Members from both taxonomic groups are likely involved in primary organic carbon degradation, since some of the reconstructed genomes encode the ability to hydrolyze recalcitrant substrates, such as cellulose and chitin. Putative sodium-pumping rhodopsins were found in both a Flavobacteriaceae- and a Chitinophagaceae-related draft genome. The predicted proteomes of both the latter and a Rhodothermaceae-related draft genome were indicative of a "salt-in" strategy of osmotic adaptation. The primary catabolic and respiratory pathways shared 
among all available reference genomes of Nanohaloarchaea and our novel genome reconstructions remain incomplete, but point to a primarily fermentative lifestyle. Encoded xenorhodopsins found in most drafts suggest that light plays an important role in the ecology of Nanohaloarchaea. Putative encoded halolysins and laccase-like oxidases might indicate the potential for extracellular degradation of proteins and peptides, and phenolic or aromatic compounds.

Keywords: soda lake brines, Nanohaloarchaea, Halobacteria, Bacteroidetes, hydrolytics, cellulase, chitinase, rhodopsin

\section{INTRODUCTION}

Modern soda lakes are characterized by high salt concentrations that can reach up to saturation and a stable high $\mathrm{pH}$, typically between 9.5 and 11 (Jones et al., 1977; Tindall, 1988; Banciu and Sorokin, 2013). They occur in (semi-)arid regions world-wide where they form naturally through evaporative concentration. Their origin is not related to seawater ("athalassic"), but to seepage of groundwater that is rich in sodium ions $\left(\mathrm{Na}^{+}\right)$ and $\mathrm{CO}_{2}$, but low in calcium $\left(\mathrm{Ca}^{2+}\right)$, and magnesium $\left(\mathrm{Mg}^{2+}\right)$ ions that would otherwise precipitate the dissolved carbonates. Whereas in the water column of "thalassic" environments and inland salt lakes, the dominant soluble salt is sodium chloride, sodium carbonate/bicarbonate salts dominate in soda lake brines and create a naturally buffered haloalkaline system. Moderately saline soda lakes are believed to be amongst the most productive aquatic environments on Earth (Melack, 1981). Complete biogeochemical carbon, nitrogen and sulfur cycles can still occur in hypersaline (salinity $>5 \% \mathrm{w} / \mathrm{v}$ ) soda lakes and become only partially hampered at salt-saturating conditions (Sorokin et al., 2014, 2015a, and references therein).

For hypersaline thalassic environments with circum-neutral $\mathrm{pH}$, it is well-established from studies on solar salterns that salinity is the major environmental factor shaping planktonic prokaryotic community composition (e.g., Rodriguez-Valera et al., 1985; Oren, 1994; Casamayor et al., 2000, 2002; Benlloch et al., 2002; Ghai et al., 2011; Dillon et al., 2013). Seawater is led into an interconnected pond system where salinity gradually increases by evaporative concentration until sea salt can be harvested. Along the pond system, proteobacterial planktonic diversity decreases in favor of extreme halophilic Archaea, such as Haloquadratum sp. belonging to the class Halobacteria (phylum Euryarchaeota) and members of the candidate class Nanohaloarchaea (phylum Nanohaloarchaeota; Ghai et al., 2011; Narasingarao et al., 2012; Martínez-García et al., 2014), as well as some moderate and extreme halophilic Bacteria such as the Gammaproteobacteria Spiribacter salinus (López-Pérez et al., 2013; León et al., 2014), Halovibrio and Halospina (Sorokin et al., 2006), and Salinibacter ruber in the Bacteroidetes phylum (Antón et al., 2000, 2002; Mongodin et al., 2005).

A similar influence of prevailing salt concentrations on microbial community composition could be inferred from previous culture-independent studies of alkaline soda lake brines. Various bacterial genera belonging to the Alphaproteobacteria, Firmicutes, Gammaproteobacteria,
Bacteroidetes, and Cyanobacteria are found in soda brines with salinities up to $250 \mathrm{~g} / \mathrm{L}$ (Humayoun et al., 2003; Mesbah et al., 2007; Dimitriu et al., 2008; Pagaling et al., 2009; Asao et al., 2011; Lanzen et al., 2013), while above these salinities Euryarchaeota belonging to the Halobacteria and the Methanosarcinales are found to dominate in natural lakes (Grant et al., 1999; Ochsenreiter et al., 2002; Mesbah et al., 2007; reviewed by Sorokin et al., 2014, 2015a) and in artificial soda concentration ponds (Gareeb and Setati, 2009; Simachew et al., 2015).

Most culture-independent studies performed in these environments world-wide are in agreement that soda lakes harbor a surprisingly high $16 \mathrm{~S}$ rRNA diversity and abundance of still uncharacterized microbes. At high salinities, the high diversity within several genera and species of the class Halobacteria is not in proportion to the relatively low number of soda lake isolates obtained from this group of mostly aerobic heterotrophs. Only recently the first alkaliphilic members of the Halobacteria capable of growing on cellulose and chitin were isolated from various soda lakes, showing that these organisms can fulfill an environmental role as primary organic matter degraders (Sorokin et al., 2015d). It is well-established, and has received considerable interest from the biotechnologyindustry, that many members of the Halobacteria are able to degrade proteins and peptides via extra-cellular proteases, also referred to as "halolysins" (De Castro et al., 2006). Almost all of these halolysins are subtilisin-like serine proteases (peptidase subfamily S8A) and quite a few are isolated, including from alkaliphilic members of the Halobacteria. Many members of the phylum Bacteroidetes are also able to consume biopolymers, such as chitin, cellulose, xylan and proteins, and are therefore often viewed as a key group for organic matter remineralization in the marine environment (e.g., Bauer et al., 2006). Interestingly, with the exception of Natronoflexus pectinovorans (phylum Bacteroidetes), polysaccharide-degrading Bacteria isolated from soda lakes belong mostly to the Firmicutes (genera Bacillus, Clostridium), Actinobacteria (genera Cellulomonas and Dietzia), Gammaproteobacteria (Alkalimonas, Marinimicrobium), and Fibrobacteres (genus Chitinovibrio; Sorokin et al., 2014, 2015a).

With the advent of next generation sequencing, direct metagenomic approaches have been successfully applied to extreme environments harboring relatively low-diversity communities. They have proven very useful to construct snapshots of the overall microbial community structure and to aid in the reconstruction of draft or even complete genomes of uncultured microorganisms (e.g., Tyson et al., 2004; Ghai 
et al., 2011; Narasingarao et al., 2012; Sharon and Banfield, 2013; Fernández et al., 2014a,b). Notably, nanohaloarchaeal $16 \mathrm{~S}$ rRNA sequences were first recovered from the hypersaline soda lake Magadi in the east-African Rift Valley (Grant et al., 1999) and since then four near-complete genomes belonging to the "Candidatus Nanohaloarchaea" have been reconstructed from metagenomes of various hypersaline aquatic habitats (Ghai et al., 2011; Narasingarao et al., 2012; Martínez-García et al., 2014). Based on $16 \mathrm{~S}$ rRNA phylogeny of sequences recovered from hypersaline and haloalkaline habitats worldwide, Nanohaloarchaea were first viewed as a deep-branching sistergroup of the class Halobacteria within the phylum Euryarchaeota (Narasingarao et al., 2012; Oren, 2014). Based on the recent genome reconstructions however, a new candidate phylum "Nanohaloarchaeota" within a "DPANN" super-phylum was proposed (Rinke et al., 2013; Castelle et al., 2015), but this view remains controversial since the outcome of archaeal phylogenetic analyses appears to depend on the choice of marker genes and reference genomes, among other factors (Petitjean et al., 2015). In addition to the absence of cultured isolates from this class and their disputed phylogeny, the lifestyle and ecological function of Nanohaloarchaea remains enigmatic.

Here we determined for the first time the overall structure of microbial communities from the brines of three natural hypersaline soda lakes and a trona crystallizer located in the Kulunda Steppe (Altai, Russia), covering a salinity range between 170 and $400 \mathrm{~g} / \mathrm{L}$ with a combination of both 16S rRNA amplicon and direct metagenome sequence analyses. In the past 15 years, these lakes have been intensively studied by classical microbiology approaches providing a good background for the interpretation of the metagenomics results. We reconstructed several draft genomes of yet uncultured populations within the phylum Bacteroidetes, class Halobacteria, and candidate class Nanohaloarchaea in order to explore their metabolic potential with respect to organic carbon remineralization in soda brines and other putative roles in biogeochemical cycling.

\section{MATERIALS AND METHODS}

\section{Site Description, Sample Collection and DNA Extraction}

The four sampled soda lakes are located in the southern part of the Kulunda Steppe, South-Western Siberia (Altai region), Russia. The exact soda lake locations, chemical parameters and general appearance of the brine at the time of sampling are given in Table 1. The eutrophic lakes Bitter-1 (B1), Tanatar trona crystallizer (Tc), and Tanatar-5 (T5) are part of the Bitter and Tanatar systems, respectively, and were formed on tertiary depositions of trona-cemented sands (Isachenko, 1951). The oligotrophic Picturesque Lake (PL) is located in the depression of the Salt Lake Steppe (sediments - gray kaolinite clays). All lakes are hypersaline, shallow ponds subjected to a very unstable water regime and with temperature fluctuations from +35 to $-35^{\circ} \mathrm{C}$ (Foti et al., 2008). At the time of sampling the salt concentration ranged from 170 to $400 \mathrm{~g} / \mathrm{L}$ sodium carbonates/chloride and a $\mathrm{pH}$ from 9.5 to 10.3 in different lakes.
Brine samples were collected in sterile glass bottles in July 2010 and 2011 and prefiltered (Whatman "White Ribbon" paper filters) to remove suspended sediment particles and aggregates. Cells from $1 \mathrm{~L}$ brine were pelleted by high speed centrifugation $(30 \mathrm{~min}$ at $13,000 \mathrm{rpm}$ with a Sorvall SLC-1500 rotor) and resuspended in $2-4 \mathrm{M} \mathrm{NaCl}$. Smaller volumes were finally pelleted $(10 \mathrm{~min}$ at $20,817 \times \mathrm{g})$ in $2 \mathrm{~mL}$ Eppendorf tubes and stored at $-70^{\circ} \mathrm{C}$ until further processing. High molecular weight DNA was extracted with the classical phenol/chloroform method (Marmur, 1961) and isopropanol precipitation after resuspending the pellets in $10 \mathrm{~mL} 0.1 \mathrm{M}$ Tris $(\mathrm{pH} 8) / 10 \mathrm{mM}$ EDTA and pretreatment with lysozyme, SDS, proteinase $\mathrm{K}$, and RNase. The quality and quantity of the extracted DNA was checked by agarose gel electrophoresis using standard markers of known concentration.

\section{Amplicon Sequencing and Analysis}

The V6-V8 region of $16 \mathrm{~S}$ rRNA was amplified using primers 926F (5' - cct atc ccc tgt gtg cct tgg cag tct cag AAA CTY AAA KGA ATT GRC GG- $\left.3^{\prime}\right)$ and 1392R ( $5^{\prime}$ - cca tct cat ccc tgc gtg tct ccg act cag - <XXXXX $>$ - ACG GGC GGT GTG TRC - 3') universal to both Archaea and Bacteria (Tremblay et al., 2015). Primer sequences were modified by the addition of $454 \mathrm{~A}$ or $B$ adapter sequences. In addition, the reverse primer included a 5 bp barcode for multiplexing of samples during sequencing. Twenty-microlitre PCR reactions were performed in duplicate and pooled to minimize PCR bias using $0.4 \mu \mathrm{l}$ Advantage GC 2 Polymerase Mix (Advantage-2 GC PCR Kit, Clontech), $4 \mu \mathrm{l}$ 5X GC PCR buffer, $2 \mu 15$ M GC Melt Solution, $0.4 \mu 110$ mM dNTP mix (MBI Fermentas), $1.0 \mu \mathrm{l}$ of each $25 \mathrm{nM}$ primer, and $10 \mathrm{ng}$ sample DNA. The thermal cycler protocol was $95^{\circ} \mathrm{C}$ for $3 \mathrm{~min}$, 25 cycles of $95^{\circ} \mathrm{C}$ for $30 \mathrm{~s}, 50^{\circ} \mathrm{C}$ for $45 \mathrm{~s}$, and $68^{\circ} \mathrm{C}$ for $90 \mathrm{~s}$, and a final $10 \mathrm{~min}$ extension at $68^{\circ} \mathrm{C}$. PCR amplicons were purified using SPRI Beads (Beckman Coulter) and quantified using a Qubit fluorometer (Invitrogen). PCR products were diluted to $10 \mathrm{ng} / \mu \mathrm{l}$ and mixed in equal concentrations. Emulsion PCR and sequencing of the PCR amplicons were performed following the Roche 454 GS FLX Titanium technology manufacturer's instructions. Sequencing tags were analyzed using the software tools PyroTagger (http://pyrotagger.jgi-psf.org) and MacQIIME (http://www.wernerlab.org/software/).

\section{Metagenome Sequencing and Raw Read Analysis}

Environmental DNA (500 ng) was sheared using the Covaris E210 and size selected for $270 \mathrm{bp}$ fragments using Agencourt Ampure Beads (Beckman Coulter) prior to library construction with the KAPA Illumina library creation kit (KAPA Biosystems). Each library was quantified by qPCR and sequenced on one lane of the Illumina HiSeq 2000 platform to generate pairedend 150 bp reads. Low quality regions of the paired-end raw reads were trimmed using DynamicTrim (Cox et al., 2010). Toplevel taxonomic profiles of the metagenomes were determined according to Ghai et al. (2011). Candidate 16S rRNA encoded metagenomic reads were initially selected by comparing a subsample of 2 million sequence pairs with a small manuallycurated database. Reads with a minimum alignment length of 
TABLE 1 | General features of the sampled soda lakes, the obtained metagenomic raw sequence reads, and the assembled contigs.

\begin{tabular}{|c|c|c|c|c|}
\hline Lake & Lake Tanatar-5 (T5) & Picturesque Lake (PL) & Lake Tanatar trona crystallizer (Tc) & Lake Bitter-1 (B1) \\
\hline Location & 51.62 N 79.84 E & 51.73 N 79.87 E & 51.65 N 79.75 E & 51.67 N $79.91 E$ \\
\hline Sampling year & 2010 & 2010 & 2011 & 2010 \\
\hline Salinity (g/L) & 170 & 250 & 300 & 400 \\
\hline pH brine & 9.9 & 9.5 & 9.9 & 10.2 \\
\hline $\mathrm{Na}_{2} \mathrm{CO}_{3}$ alkalinity $(\mathrm{M})$ & 0.8 & 1.1 & 4.0 & 2.0 \\
\hline Total carbonate alkalinity (M) & 1.9 & 2.8 & 5.0 & 4.4 \\
\hline Brine appearance & Muddy & Muddy & Orange-red & Oily yellow \\
\hline Metagenome ID & $\mathrm{T} 5-\mathrm{Br} 10$ & PL-Br10 & Tc-Br11 & $\mathrm{B} 1-\mathrm{Br} 10$ \\
\hline SRA Accession & INGZ-SRA052017 & INHF-SRA052008 & INHA-SRA052024 & INHB-SRA052018 \\
\hline Number of read pairs & $206,674,382$ & $352,665,522$ & $191,945,135$ & $411,685,143$ \\
\hline Estimated mean coverage & $140 x$ & $190 x$ & $129 x$ & $202 x$ \\
\hline Number of assembled contigs & 636,016 & $1,140,049$ & 458,620 & 482,919 \\
\hline Number of contigs $\geq 5 \mathrm{~kb}$ & 19,350 & 25,098 & 9,426 & 15,551 \\
\hline GenBank Accession (contigs $\geq 5$ kb) & LFIK00000000 & LKMJ00000000 & LFFM00000000 & LFCJ00000000 \\
\hline Average contig length (b) & 1,005 & 974 & 895 & 1,115 \\
\hline Maximum contig length (b) & 204,877 & 192,059 & 134,932 & 155,378 \\
\hline Total assembly length (b) & $639,293,147$ & $1,110,081,880$ & $410,404,879$ & $538,272,225$ \\
\hline
\end{tabular}

Raw sequence reads have been deposited to the NCBI Sequence Read Archive (SRA) and contigs larger than $5 \mathrm{~kb}$ at DDBJ/EMBL/GenBank.

90 bases (min identity $80 \%, e$-value $1 \mathrm{e}-5$ ) were extracted and compared to sequences in the Ribosomal Database Project (Cole et al., 2014). The best-named hit was used to classify the reads to top level taxa. Chloroplast $16 \mathrm{~S}$ rRNA was manually filtered out and taxa containing less than $1 \%$ of the total assigned $16 \mathrm{~S}$ rRNA reads were not considered.

\section{Reconstruction and Analysis of Draft Genomes}

Assembly of the metagenomic reads was performed with MEGAHIT v1.0.1-13-gd6932f9 (Li et al., 2015). Protein coding genes on contigs longer than $5 \mathrm{~kb}$ were predicted with Prodigal (Hyatt et al., 2010), tRNAs with tRNASCAN (Lowe and Eddy, 1997), rRNAs using meta_rna (Huang et al., 2009) and annotated using BLAST against the non-redundant database Pfam (Bateman et al., 2004), COGs (Tatusov et al., 2003), and TIGRfams (Haft et al., 2001). Genes at the end of contigs and incomplete genes without valid start/stop codons were removed. Based on sequence similarity against the non-redundant NCBI database, the best hit for each gene was determined and used to bin to top level taxa. Only those contigs that contained a minimum of three genes and had not more than half of the genes hitting different taxa were retained. Further binning of the contigs belonging to Bacteroidetes, Nanohaloarchaea, or Halobacteria was done by differential coverage binning with CONCOCT (Alneberg et al., 2014) after using subsets of 20 million sequence reads of each dataset to estimate coverage by mapping reads with min $95 \%$ nucleotide identity and $50 \mathrm{bp}$ overlap. Possible bin contamination and strain heterogeneity were estimated with CheckM v0.9.7 (Parks et al., 2014) and only those with reasonable concatenated length $(>0.5-0.8 \mathrm{Mb})$ and with contamination below $5 \%$ were retained for subsequent analysis.
Taxonomic affiliation of the selected bins was determined via phylogenetic trees based on concatenates of all proteins identified within Clusters of Orthologous Groups (COG; Tatusov et al., 2003) and shared with complete or near-complete reference genomes (RefSeq NCBI, plasmids excluded), since no $16 \mathrm{~S}$ rRNA genes were found on the contigs. Shared proteins were concatenated and aligned using Kalign (Lassmann and Sonnhammer, 2005) and a maximum-likelihood tree was made using the software program MEGA version 6 (Tamura et al., 2013). The completeness of the reconstructed bacterial and archaeal genomes was assessed based on the presence of 112 essential genes (Albertsen et al., 2013) and 53 core genes (Narasingarao et al., 2012) respectively, using HMMER. Using an $e$-value cut-off of $1 \mathrm{e}-5$, lower and upper bound predictions of completeness were obtained using all predicted genes or only the best annotations (hcov and qcov $>80 \%$ ). Average nucleotide identity (ANI) and conserved DNA fraction between reconstructed and/or reference genomes were calculated based on the whole genome sequence as described by Goris et al. (2007).

The carbon-, nitrogen- and sulfur metabolic potential of the draft genomes was inferred from all proteins identified within COG, as well as from KEGG pathways and BRITE hierarchies after re-annotating the predicted proteins from the standard draft genomes automatically using KAAS (KEGG Automatic Annotation Server: http://www.genome.jp/kegg/kaas/; Moriya et al., 2007). Additionally, assigned K-numbers were used to screen for the presence of functional marker genes (Lauro et al., 2011; Llorens-Marès et al., 2015). Putative encoded carbohydrate-active enzymes in the draft genomes were identified by sequenced-based annotation of the predicted proteins from all coding sequences using the CAZymes Analysis Toolkit (CAT; Park et al., 2010; Lombard et al., 2014). 
To determine the general mode of osmotic adaptation of the putative microorganisms for which we reconstructed the genomes, the isoelectric points ( $\mathrm{pI}$ ) of the coding sequences with a minimum length of 30 amino acids were calculated using the program IEP in the EMBOSS package (Rice et al., 2000). The pI distribution (bin width 0.1 ) for each composite genome was compared to the pI distribution of selected, complete reference genomes available in RefSeq. Genes likely to be involved in osmotic adaptation were identified through their annotations and best-hits (see above), namely those involved in osmolyte synthesis and uptake or those encoding putative ion transporters and channels.

\section{Read Recruitments}

Population abundance in the different soda lake brines was estimated via read recruitments to the draft genomes from subsamples of 20 million reads from the different metagenomic datasets. A cut-off of $95 \%$ identity in at least 50 bases was used and the number of hits was normalized against the size of the genomes and metagenomes and expressed as Reads Per Kilobase of sequenced reads per Gigabase of mapped reads (RPKG; LópezPérez et al., 2013).

\section{Data Availability}

The raw sequence reads of the four metagenomics datasets have been deposited to the NCBI Sequence Read Archive (SRA; Accession Numbers given in Table 1). The assembled contigs larger than $5 \mathrm{~kb}$ have been deposited as Whole Genome Shotgun projects at DDBJ/EMBL/GenBank (Accession Numbers given in Table 1). The reconstructed draft genomes have been deposited as Whole Genome Shotgun projects at DDBJ/EMBL/GenBank under the accessions LKMK00000000LKNH00000000 (complete list given in Supplementary Table 2 ). All versions described in this paper are version XXXX010 00000.

\section{RESULTS AND DISCUSSION}

\section{Microbial Community Composition Based on 16S rRNA Gene Sequences}

Top Level Taxa Abundance in Relation to Salinity

Microbial community composition of the different soda lake brines was compared based on metagenomic reads containing fragments of the 16S rRNA gene as well as on OTUs assigned to the amplicon sequences (Figure 1). The majority of the selected metagenomic reads originating from the saturated salt brines (total salinity $\geq 250 \mathrm{~g} / \mathrm{L}$ ) of Bitter-1 (B1), Tanatar trona crystallizer (Tc), and Picturesque Lake (PL) belonged to the archaeal phylum Euryarchaeota (>91\%). Those from Tanatar-5 (T5) with a total salinity of $170 \mathrm{~g} / \mathrm{L}$ belonged to the bacterial taxa Bacteroidetes (34\%), Gammaproteobacteria (33\%), and Alphaproteobacteria (12\%) and to a lesser extent to the Actinobacteria, Firmicutes, Cyanobacteria, and unclassified bacterial groups $(<5 \%)$. Euryarchaeota still constituted a considerable fraction of the prokaryotic community in the brine of T5 (7\%). Similarly, 16S rRNA gene amplicon sequences belonging to Euryarchaeota dominated in the three most saline brine datasets, while those belonging to Bacteria dominated the lower salinity dataset. Our results are in agreement with previous culture-independent studies that reported the dominance of Euryarchaeota in 16S rRNA gene clone libraries from hypersaline soda lakes (Grant et al., 1999; Ochsenreiter et al., 2002; Mesbah et al., 2007; Simachew et al., 2015). Moreover, all bacterial top level taxa that were previously found to be common in soda lakes world-wide with a maximum salinity of $250 \mathrm{~g} / \mathrm{L}$ (Humayoun et al., 2003; Mesbah et al., 2007; Dimitriu et al., 2008; Pagaling et al., 2009; Asao et al., 2011; Lanzen et al., 2013) appeared abundant $(>1 \%)$ in both our metagenomic and amplicon T5 dataset.

Amplicon and shotgun sequence-based taxonomic profiling introduced different biases. While the direct metagenomic approach might have left rare taxa undetected (Lynch and Neufeld, 2015), amplification biases in complex DNA mixtures might have distorted the original 16S rRNA gene ratios. Most evidently for the three most saline lakes, rare (here $<1 \%$ ) bacterial taxa were overrepresented in the amplicon datasets compared to the metagenomes, while archaeal $16 \mathrm{~S}$ sequences were underrepresented. Furthermore, between 16 and $23 \%$ of the $16 \mathrm{~S}$ amplicon sequences from the brines of B1, PL and T5 could not be assigned to any top level bacterial or archaeal taxon ("unclassified"). Since no "unclassified" sequences were found in taxonomic profiles derived from the metagenomes, these are suspected to originate from eukaryote organelles other than chloroplasts. Cyanobacteria were absent in the three most saline brines when considering the directly sequenced metagenomes, but present in the same brines when considering the amplicon sequences. Finally, on the phylum and class level, the total number of detected taxa (including "Unclassified" Bacteria and Archaea) in the metagenomes was inversely related to salinity with six taxa detected for B1 (400 g/L salt) and 14 for T5 (Supplementary Table 3). This trend was not apparent in the amplicon sequencing datasets where the brines of PL and T5 appeared the most and the least rich (19 and 11 top level taxa OTUs), respectively.

\section{Cultured vs. Unknown Members of the most Abundant Top Level Taxa}

While the directly sequenced, short-read metagenomes yielded in our opinion more realistic snapshots of the relative 16S rRNA gene abundances of the high-level community constituents, we considered only the longer amplicon sequences $(\sim 450 \mathrm{nt}$ vs. $<150 \mathrm{nt}$ ) for further phylogenetic assignment down to the genus-level, focusing on the identified abundant top-level taxa (Table 2).

\section{Euryarchaeota}

More than $99 \%$ of the OTUs assigned to Euryarchaeota in the three amplicon sequencing datasets from the three most saline brines of PL, Tc, and B1, were affiliated with 11 distinct and known genera from the class Halobacteria. Among them, Halorubrum - and Natrinema-related sequences were the most abundant ones. The remaining largest fraction of the OTUs, as well as all Halobacteria-related sequences from the brine of T5, could not be assigned to any known genus. 


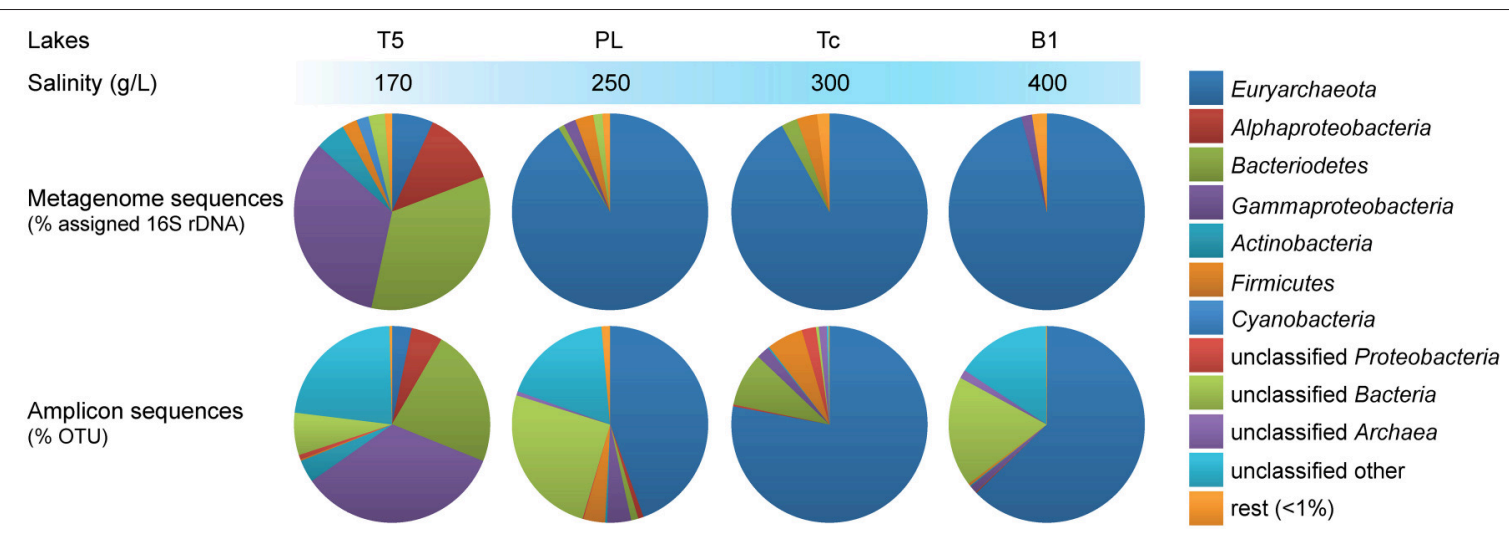

FIGURE 1 | Taxonomic profiles of the microbiota from hypersaline soda lake brines. Top: distribution of sampled metagenomic reads encoding fragments of 16S rRNA genes assigned to top level taxa. Bottom: distribution of the OTUs assigned to the amplicon sequences. T5, Tanatar-5; PL, Picturesque Lake; Tc, Tanatar trona crystallizer; B1, Bitter-1.

Few Methanosarcinaceae-related sequences of the genus Methanolobus (class "Methanomicrobia") were found in the brine of T5 (1.2\% of Euryarchaeota-related OTUs or $0.04 \%$ of the total OTUs-see Table 2). The presence of this specific genus of moderately salt-tolerant, methylotrophic methanogens was also detected in $16 \mathrm{~S}$ clone libraries obtained from the hypersaline lake Fazda (Wadi an Natron, Egypt; Mesbah et al., 2007). More recently, Methanolobus was also found in the sediments of hypersaline soda lakes of the Kulunda Steppe by a functional gene assay (Sorokin et al., 2015b). In addition, two Methanolobus isolates were obtained and potential methanogenic activity up to soda saturating conditions confirmed. Although methanogens are typically sediment-affiliated and suspended clay particles might have passed the pre-filtration step in this study, oxygen solubility in hypersaline brines is low (e.g., Grant, 2004) and therefore Methanolobus-related sequences might also originate from methanogens thriving in the brine of T5 under anoxic conditions.

\section{Bacteroidetes}

Most of the Bacteroidetes OTUs in T5 belonged to the genus Gracilimonas ( $\sim 83 \%)$. This genus comprises a group of marine (Choi et al., 2009; Cho et al., 2013) and salt mine (Wang et al., 2013) isolates and related $16 \mathrm{~S}$ gene fragments were recovered recently from a haloalkaline soil (Keshri et al., 2013). In the datasets of the more saline brines of B1, Tc, and PL, Gracilimonasrelated sequences were also the dominant Bacteroidetes. The two important remaining Bacteroidetes fractions in the brine of T5 were affiliated with unknown genera from the families Chitinophagaceae ( $11 \%$ ) and Flavobacteriaceae ( $5 \%)$.

\section{Alphaproteobacteria}

From the OTUs assigned to the Alphaproteobacteria in the T5 dataset, the dominant fraction $(\sim 63 \%)$ was affiliated with the family Rhodobacteraceae (order Rhodobacterales) and belonged to the genera Roseinonatronobacter, Rhodobacter and Rhodobaca. The genus Roseinonatronobacter comprises the haloalkaliphilic members of a unique group of aerobic, bacteriochlorophyll a-containing, sulfur-oxidizing lithoheterotrophs that were first isolated from soda lake microbial mats (Sorokin et al., 2000) and later from the low-salt epilimnion of the alkaline Mono Lake (Boldareva et al., 2007). Haloalkaliphilic members of the purple non-sulfur bacteria belonging to the latter two genera are frequently detected in soda lakes (Rees et al., 2004; Kompantseva et al., 2007; Asao et al., 2011). While members of the genus Rhodobaca were isolated from soda lakes (Milford et al., 2000; Boldareva et al., 2008), so far no alkaliphilic Rhodobacter isolates are obtained. Most of the Rhodobacteraceae-related OTUs from T5 could not be assigned to a known genus, which was also a conclusion from previous research on Kulunda soda lakes that focused on this group (Kompantseva et al., 2010). Another large alphaproteobacterial fraction $(\sim 32 \%)$ was assigned to the order Rhizobiales, but no family could be assigned. The presence of the rhizobial genus Mesorhizobium was reported previously in the sediment and dry mud from a Kenyan soda lake (Mwirichia et al., 2011) and in a recent molecular survey of Kulunda soda lake sediments (Tourova et al., 2014). Although there is a high probability that the Rhizobiales-related sequences in this study are from terrestrial origin, the order includes, besides plant rhizobia, plant- and animal-pathogens, photosynthetic purple non-sulfur bacteria that occur in soda lakes, such as the genera Rhodopseudomonas, Rhodoblastus, and Rhodomicrobium (Tourova et al., 2011). In the datasets of the more moderate saline brines of PL and T5, a substantial fraction of the alphaproteobacterial OTUs affiliated with the Rhodospirillaceae, another phylogenetic group of purple nonsulfur bacteria (Imhoff, 1995), still without soda lake isolates.

\section{Gammaproteobacteria}

From the OTUs assigned to the Gammaproteobacteria in the T5 dataset, the dominant fraction $(\sim 59 \%)$ was affiliated with no known order; the largest remaining fractions belonged to the genera Thioalkalivibrio (order Chromatiales; $~ 37 \%$ ) and Halomonas (order Oceanospirillales; $\sim 2 \%$ ), comprising haloalkaliphilic sulfur-oxidizing bacteria and aerobic heterotrophs commonly occurring in soda lakes with various salinities 


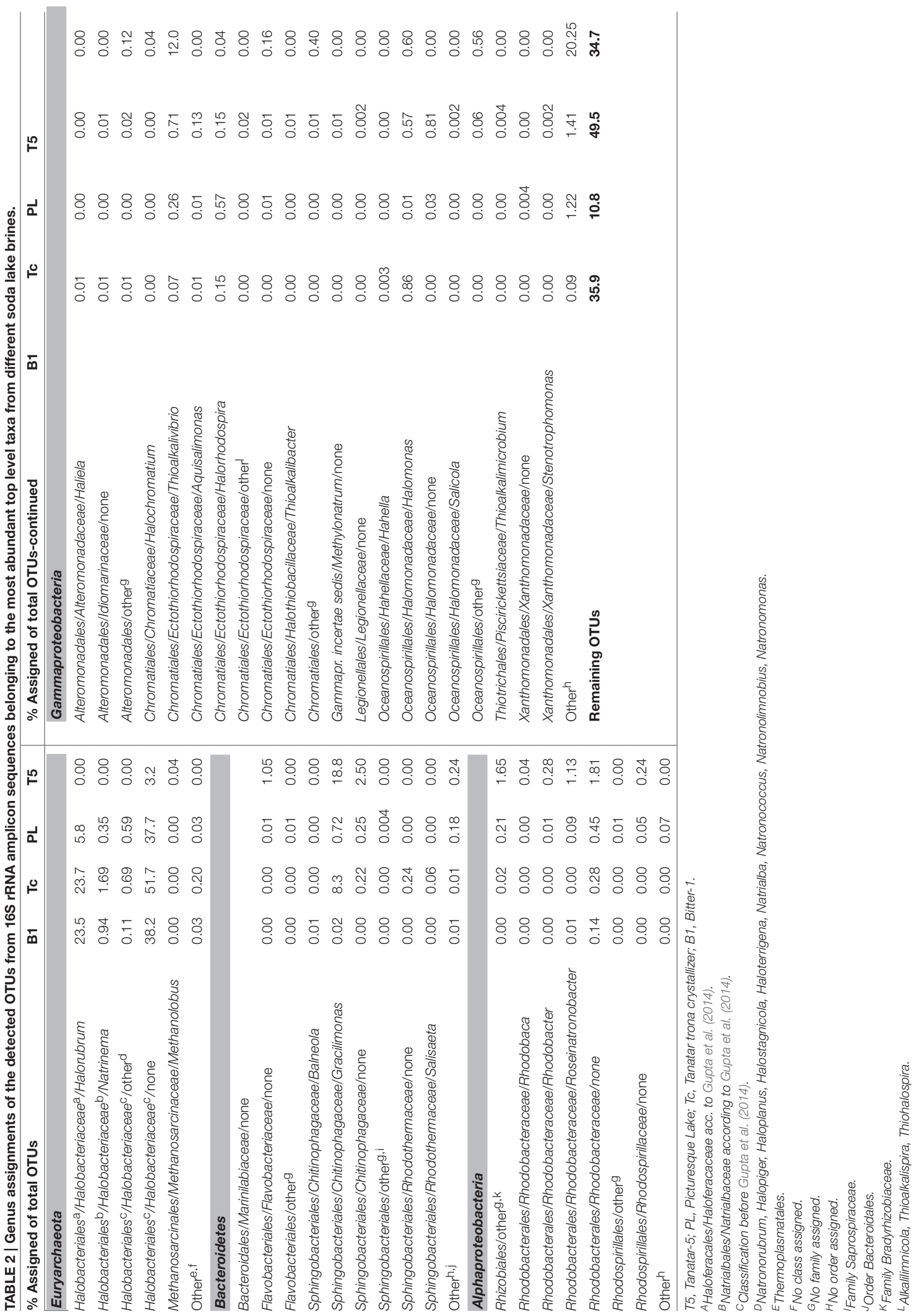


(Sorokin et al., 2014). In contrast, from the OTUs assigned to the Gammaproteobacteria in the dataset B1, the soda lake with the highest salinity, the dominant fractions belonged to Halomonas $(\sim 71 \%)$ and purple sulfur bacteria (genus Halorhodospira, order Chromatiales; $\sim 13 \%)$.

\section{Draft Genomes of Putative Predominant, Yet Still Uncultured Community Members}

After assembling the metagenomic reads into contigs and classifying all $\geq 5 \mathrm{~kb}$ contigs to top-level taxa, we obtained several high-quality $(<4 \%$ estimated contamination using CheckM) draft genomes of uncultured members of the Euryarchaeota, Bacteroidetes and the candidate class Nanohaloarchaea (Table 3), as well as a large number of contigs belonging to Eukaryota, Gamma- and Alphaproteobacteria and Actinobacteria (Supplementary Table 1). The obtained draft genomes represent "population" genomes i.e., not clonal (Sharon and Banfield, 2013) of single species or strains. Below we discuss their inferred phylogeny based on genome trees (Figures 2A-E) and average nucleotide identities (ANIs; Supplementary Tables 4-8) with known organisms, their metabolic potential and principal modes of osmotic adaptation (Figure 3).

\section{Candidate Class Nanohaloarchaea Phylogeny}

From B1-Br10 and PL-Br10 we obtained eight draft genomes belonging to low-GC Archaea of the candidate class Nanohaloarchaea (Table 3; Figure 2A; Supplementary Table 4). Based on the genome tree, four of these reconstructions, two from each brine, were most closely related to the draft genome of "Candidatus Haloredivivus" obtained from metagenomes in a solar saltern pond (Ghai et al., 2011). They belonged to four different genera based on average nucleotide identity (all ANIs $<74.9 \%$ ). The remaining four draft genomes belonged to one to three different genera, distantly related to "Candidatus Nanosalina" obtained from a hypersaline lake (Narasingarao et al., 2012; all ANIs < 74.5\%), while the drafts B1-Br10-U2g1 and PL-Br10-U2g19 belonged to the same species thriving at different brines (ANI $=99.4 \%)$.

\section{Xenorhodopsins and heterotrophy}

We found several genes that support the predominant heterotrophic lifestyle previously proposed for "Candidatus Haloredivivus" (Ghai et al., 2011; Narasingarao et al., 2012). Notably, out of the eight draft genomes, six encoded putative xenorhodopsins for which the exact physiological role still remains enigmatic (Ugalde et al., 2011; Supplementary Figure 1, Supplementary Table 9). Starch-degrading metabolism was evident from encoded alpha-amylases and related glucoside hydrolases (CAZy families GH13 and GH57) found in seven out of eight drafts. In five drafts subtilisin-like proteins (peptidase S8) were encoded, suggesting degradation potential for extracellular proteins and peptides. Furthermore, in B1-Br10_U2g21 and B1-Br10_U2g19 we found laccase-like Cu-oxidases encoded that may be involved in the breakdown of phenolic compounds, ring cleavage of aromatic compounds or lignin (Ausec et al., 2011). A near-complete Embden-Meyerhoff pathway was encoded in all drafts, which include some typical archaeal enzymes such as an ADP-dependent phosphofructokinase/glucokinase (EC 2.7.1.147) and a 2,3-bisphosphoglycerate-independent phosphoglycerate mutase (EC 5.4.2.12; Siebers and Schönheit, 2005). The draft genomes also encoded a "classical" glucose-6phosphate isomerase as found in most Eukarya and Bacteria. Moreover, they encoded an eukaryal-type Class I fructose1,6-biphosphate aldolase (EC 4.1.2.13), which is unusual in Archaea, only shared with some members of the Halobacteria and Nanoarchaeota (Siebers et al., 2001; Say and Fuchs, 2010). The potential for conversion of pyruvate to acetyl CoA was encoded by a pyruvate dehydrogenase complex, while a pyruvate:ferredoxin oxidoreductase (pyruvate synthase; EC 1.2.7.1) was only encoded in the reference genome of "Candidatus Nanosalinarum."

\section{Fermentative lifestyle}

Several genes related to central carbon metabolism and energy production were present in our draft genomes and the four publicly available drafts of Nanohaloarchaea described by Ghai et al. (2011), Narasingarao et al. (2012), and Martínez-García et al. (2014) suggest an anaerobic fermentative lifestyle, which contrasts to the principally aerobic respiratory metabolism suggested previously for "Candidatus Nanosalina" and "Candidatus Nanosalinarum" by Narasingarao et al. (2012). First, we found an ADP-forming acetyl-CoA synthase (EC 6.2.1.13), typical for acetate-forming anaerobic fermentative Archaea and facultative anaerobic Halobacteria (Siebers and Schönheit, 2005) as well as a malic enzyme (EC 1.1.1.38/39), a lactate dehydrogenase (the latter was also reported by Narasingarao et al., 2012), ferredoxin and a pyruvate:ferrodoxin oxidoreductase which suggests at least the potential for incomplete fermentation. Second, while most draft genomes encoded a $\mathrm{H}^{+}$-translocating NADH:ubiquinone reductase (EC 1.6.5.3), a plastocyanin-like type I copper protein (most likely involved in electron transfer reactions, e.g., Martin-Cuadrado et al., 2015) and V-type ATPases, none contained identifiable aerobic terminal oxidases. Andrade et al. (2015) most recently reported the absence of cytochrome $c$ oxidases in novel Nanohaloarchaea-related draft genomes as well. Finally, in contrast to Narasingarao et al. (2012) we did not find a complete or reduced TCA-cycle in any of the available drafts, only a putative citrate synthase (EC 2.3.3.1) and both subunits of a putative succinyl CoA synthetase (EC 6.2.1.5), as well as a putative citryl CoA ligase (EC 4.1.3.34). In the available genome of "Candidatus Haloredivivus" however, the succinyl CoA synthetase was annotated as a citryl-CoA synthetase and had $35 \%$ sequence identity (Blast-P; e-value 4e-66) to the citryl-CoA synthetase of Hydrogenobacter thermophilus (bacterium phylum Aquificae). Citryl-CoA synthetase has been shown to act in the latter organism in concert with citrylCoA lyase as an alternative citrate cleavage pathway to the reductive TCA cycle (Hügler et al., 2007). Hypothetically for the Nanohaloarchaea, both enzymes, together with malic enzyme, could perhaps function in the ATP-dependent regeneration of acetyl-CoA and oxaloacetate rather than in the aerobic TCA cycle. 
TABLE 3 | Phylogenetic affiliation and general features of selected reconstructed draft genomes.

\begin{tabular}{|c|c|c|c|c|c|c|c|c|c|c|c|}
\hline Name $<$ Id $>$ & C & $\mathbf{L}$ & GC\% & ORF & tRNA & $16 S$ & $5 S$ & 235 & Cont & Str het & Compl \\
\hline Chitinophagaceae bacterium T5-Br10_B2g13 & 258 & 4.30 & 41 & 7004 & 35 & 0 & 2 & 0 & 2.1 & 0.0 & $80.2-91.9$ \\
\hline Flavobacteriaceae bacterium T5-Br10_B2g0 & 132 & 1.49 & 33 & 2494 & 21 & 0 & 0 & 0 & 0.9 & 33.3 & $61.3-71.2$ \\
\hline Rhodothermaceae bacterium Tc-Br11_B2g6_7 & 109 & 3.20 & 62 & 5220 & 38 & 1 & 1 & 1 & 1.1 & 0.0 & $79.3-92.8$ \\
\hline Halobacteriaceae archaeon Tc-Br11_E2g27 & 205 & 2.10 & 55 & 3920 & 25 & 0 & 0 & 0 & 0.4 & 0.0 & $69.3-79.2$ \\
\hline Haloferacaceae archaeon PL-Br10_E2g46 & 171 & 1.44 & 51 & 2586 & 19 & 0 & 0 & 0 & 0.5 & 25.0 & $67.9-77.4$ \\
\hline Haloferacaceae archaeon B1-Br10_E2g22 & 152 & 2.37 & 58 & 4738 & 35 & 0 & 1 & 0 & 3.2 & 35.7 & $73.6-83.0$ \\
\hline Haloferacaceae archaeon PL-Br10_E2g29 & 134 & 1.28 & 62 & 2478 & 21 & 0 & 0 & 0 & 1.6 & 44.4 & $62.3-73.6$ \\
\hline Halorubrum sp. PL-Br10_E2g5 & 160 & 1.48 & 65 & 2756 & 19 & 0 & 0 & 0 & 0.4 & 50.0 & $79.2-83.0$ \\
\hline Haloferacaceae archaeon Tc-Br11_E2g18 & 139 & 1.27 & 67 & 2372 & 15 & 0 & 0 & 0 & 0.0 & 0.0 & $62.3-75.5$ \\
\hline Nanohaloarchaea archaeon B1-Br10_U2g1 & 26 & 0.710 & 43 & 1634 & 29 & 0 & 0 & 1 & 0.0 & 0.0 & 84.9-94.3 \\
\hline Nanohaloarchaea archaeon B1-Br10_U2g21 & 24 & 0.816 & 39 & 1756 & 34 & 0 & 1 & 1 & 2.8 & 50.0 & $81.1-86.8$ \\
\hline Nanohaloarchaea archaeon B1-Br10_U2g19 & 35 & 0.663 & 41 & 1472 & 19 & 1 & 0 & 0 & 0.0 & 0.0 & $81.1-90.6$ \\
\hline Nanohaloarchaea archaeon B1-Br10_U2g29 & 35 & 0.526 & 40 & 1182 & 19 & 0 & 1 & 0 & 1.0 & 0.0 & $64.2-73.6$ \\
\hline Nanohaloarchaea archaeon PL-Br10_U2g5 & 26 & 0.84 & 41 & 1936 & 34 & 1 & 1 & 1 & 0.0 & 0.0 & $88.7-94.3$ \\
\hline Nanohaloarchaea archaeon PL-Br10_U2g19 & 55 & 0.76 & 42 & 1702 & 34 & 0 & 2 & 2 & 0.9 & 0.0 & $81.1-90.6$ \\
\hline Nanohaloarchaea archaeon PL-Br10_U2g16 & 54 & 0.65 & 42 & 1448 & 18 & 0 & 0 & 0 & 2.3 & 20.0 & $81.1-90.6$ \\
\hline Nanohaloarchaea archaeon PL-Br10_U2g27 & 52 & 0.58 & 43 & 1290 & 22 & 3 & 0 & 2 & 0.9 & 0.0 & $73.6-77.4$ \\
\hline Natrialbaceae archaeon B1-Br10_E2g2 & 251 & 2.875 & 66 & 5368 & 30 & 0 & 4 & 0 & 3.7 & 11.8 & $79.2-84.9$ \\
\hline Natrialbaceae archaeon B1-Br10_E2g27 & 207 & 2.01 & 60 & 3710 & 22 & 0 & 1 & 0 & 0.8 & 0.0 & $62.3-71.7$ \\
\hline Natrialbaceae archaeon Tc-Br11_E2g1 & 210 & 2.16 & 65 & 3990 & 27 & 0 & 0 & 0 & 1.8 & 20.0 & $75.5-84.9$ \\
\hline Natrialbaceae archaeon Tc-Br11_E2g14 & 136 & 3.16 & 63 & 5916 & 48 & 0 & 0 & 0 & 1.6 & 0.0 & $84.7-94.3$ \\
\hline Natrialbaceae archaeon Tc-Br11_E2g28 & 216 & 2.33 & 61 & 4342 & 34 & 0 & 2 & 1 & 0.3 & 0.0 & $71.7-86.8$ \\
\hline Natrialbaceae archaeon Tc-Br11_E2g8 & 192 & 2.29 & 69 & 4206 & 32 & 0 & 1 & 0 & 0.7 & 16.7 & $71.7-83.0$ \\
\hline Natrialbaceae archaeon PL-Br10_E2g26 & 167 & 1.46 & 61 & 2646 & 18 & 0 & 2 & 0 & 0.0 & 0.0 & $67.9-81.1$ \\
\hline
\end{tabular}

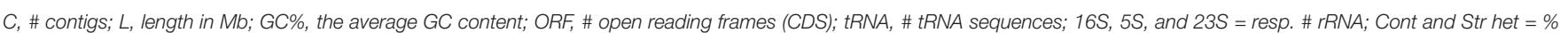

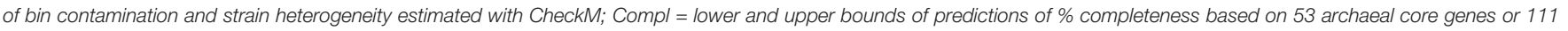
single copy genes for the bacterial drafts.

\section{Halobacteria \\ Phylogeny}

Thirteen draft genomes from members of all three known orders within the class Halobacteria (as recently defined in Gupta et al., 2014) were obtained from the datasets B1-Br10, Tc-Br11 and PL-Br10 of the most saline soda brines (Table 3). Within the family Natrialbaceae, the two drafts B1-Br10_E2g2\&27 were affiliated with the genera Natronoccoccus, Natrialba, and Natronobacterium (Figure 2B; Supplementary Table 5). Since, $16 \mathrm{~S}$ rRNA sequences belonging to these genera were found in the corresponding amplicon datasets of B1, but their ANI and the ANIs with the three complete reference genomes shown in our genome tree were only between 74.3 and $77.4 \%$, it is not clear whether the drafts belong to one of these three genera or represent one or two novel, distinct genera. Five more drafts of Natrialbaceae-related organisms were reconstructed that, based on our genome tree, appeared to be more related to each other than to any other complete genome sequenced from this family. The drafts Tc-Br11_E2g28 and PL-Br10_E2g26 belonged to the same species ( $\mathrm{ANI}=98.6 \%$ ) thriving in different brines. Within the family Haloferacaceae, the two drafts Tc-Br11_E2g18 and PL-Br10_E2g5 were affiliated with the genus Halorubrum (Figure 2C; Supplementary Table 6). Since the latter had an ANI of $80.6 \%$ with Halorubrum lacusprofundi ATCC 49239, it almost certainly belongs to the genus Halorubrum. The three drafts PL-Br10_E2g29\&46 and B1-Br10_E2g22 were affiliated with the genus Halonotius, but most likely belonged to three distinct, novel genera (ANIs between 69.9 and 74.6\%). One draft genome obtained from the trona crystallizer Tc, Tc-Br11_E2g27 belonged to the family Halobacteriaceae (Figure 2D; Supplementary Table 7) and comprised a distinct genus related to Halorhabdus $(\mathrm{ANI}=68.7 \%)$.

\section{Rhodopsins and metabolic versatility}

All reconstructed draft genomes encoded for versatile metabolisms indicating aerobic heterotrophy. Almost all draft genomes possessed a halorhodopsin and/or light-activated signal transducers (sensory rhodopsin I and II) typical of extremely halophilic Archaea (Supplementary Table 9). Most drafts encoded a near-complete Krebs cycle and the potential conversion of pyruvate to acetyl $\mathrm{CoA}$ by a pyruvate synthase or in some cases by a pyruvate dehydrogenase complex. All drafts encoded subunits I and/or III of a cytochrome $c$ oxidase most likely functioning as a terminal oxidase for aerobic respiration (Supplementary Table 10). Almost all genomes encoded a putative L-lactate dehydrogenase (EC 1.1.1.27), which might point to the ability for sugar fermentation. Notably, all of the Natrialbaceae-related drafts as well as three of the 


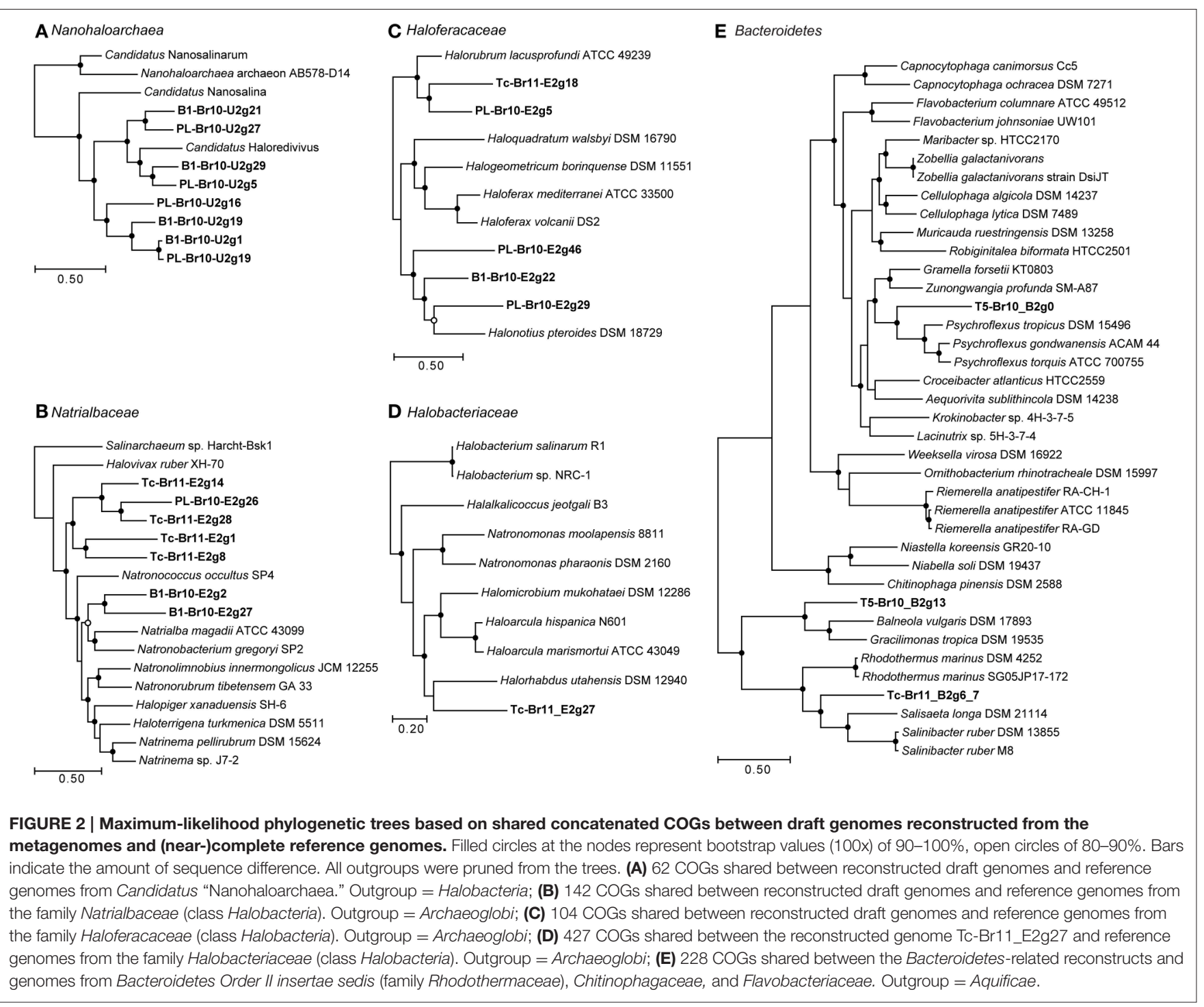

Haloferacaceae-related drafts encoded a tungsten-containing aldehyde:ferredoxin oxidoreductase (AOR; EC 1.2.7.5), a widespread enzyme amongst facultative fermenters (Hagedoorn et al., 2005). Five of the halobacterial drafts encoded the catalytic subunit PsrA of a putative molybdenum-containing thiosulfate/polysulfide reductase (PsrABC; Supplementary Table 10), which can catalyze the quinone-coupled reduction of polysulfides $\left(\mathrm{S}_{\mathrm{n}}^{2-}\right)$ in the bacterium Thermus thermophilus (class Deinococci) thriving in geothermal hot springs (Jormakka et al., 2008). Furthermore, membrane-bound polysulfide reductases were expressed during growth of Halanaeroarchaeum sulfurireducens HSR2, a sulfidogenic, extreme halophilic, neutrophilic, obligate anaerobic member of the Halobacteriaceae capable of dissimilatory reduction of elemental sulfur using acetate as the sole electron donor (Sorokin et al., 2015c). Polysulfides are unstable intermediates formed by the chemical reaction of elemental sulfur with free sulfide in the $\mathrm{pH}$-neutral environment from which $H$. sulfurireducens was obtained. In contrast, thiosulfate and polysulfides are the expected abundantly present sulfur intermediates in alkaline environments such as soda brines. Four of the five PsrABC-containing drafts also encoded rhodanese-like sulfurtransferases (EC:2.8.1.1), which are suggested to be involved in the binding, stabilization and transfer of polysulfides to polysulfide reductase in $H$. sulfurireducens HSR2 (Sorokin et al., 2015c). Additionally, Haloferacaceae archaeon Tc-Br11_E2g18 encoded the electron-transfer subunit (NarH) of a putative membrane-bound nitrate reductase (Nar). The molybdoenzyme Nar has been previously shown to function as a terminal electron acceptor in Haloarcula marismortui and Haloferax sp. (families Halobacteriaceae and Haloferacaceae respectively) under denitrifying conditions (reviewed by Cabello et al., 2004).

\section{Central carbon metabolism}

The glycolytic pathway within their central carbon metabolisms appeared to be rather versatile as well. From all three 

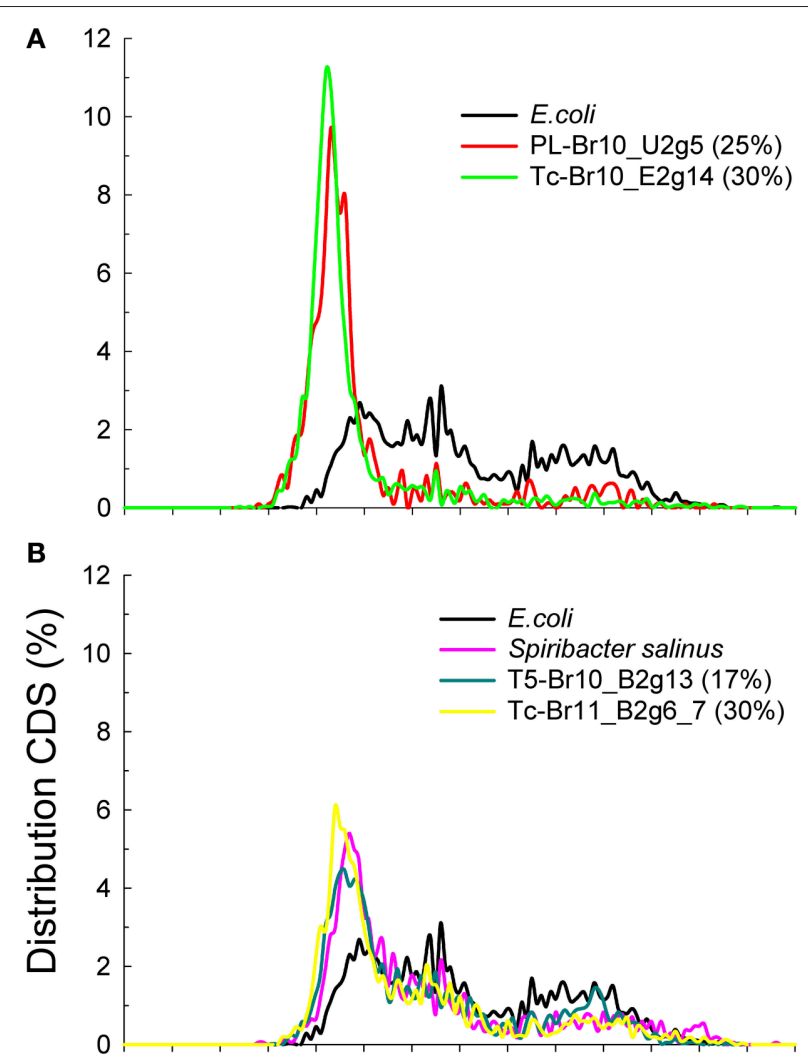

C

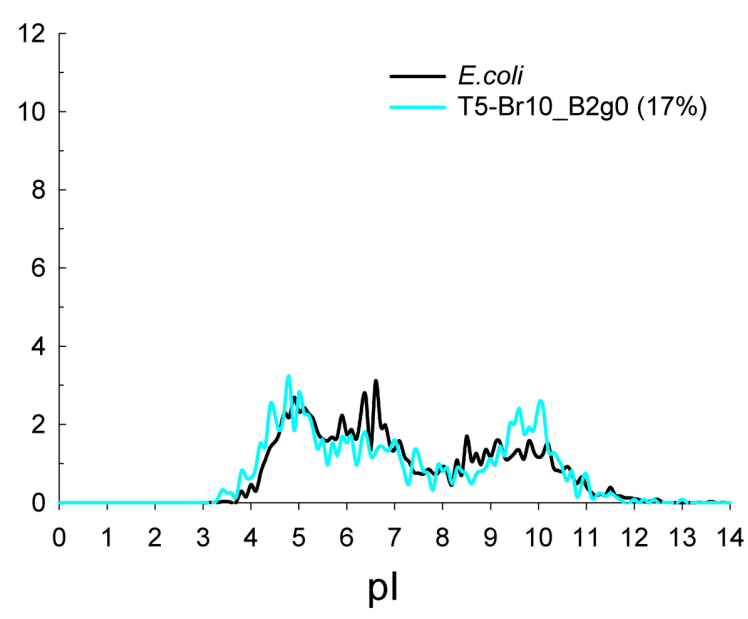

FIGURE 3 | Predicted isoelectric points (pl) for the coding sequences (CDS) of a selection of draft genomes and complete reference genomes indicative for different strategies of osmotic adaptation. (A) archaeal "salt-in": all archaeal draft genomes had a similar pl-profile, only one Nanohaloarchaea- and one Natrialbaceae-related draft genome are shown here; (B) bacterial "salt-in"; (C) "salt-out." The salinities of the brines from which the draft genomes were reconstructed are depicted in between brackets (in \%).

families draft genomes were obtained that encoded a 2keto-3-deoxygluconate kinase (KDG kinase; EC 2.7.1.45), the key-enzyme of non- or semi-phosphorylative EntnerDoudoroff pathway for glucose degradation (Siebers and Schönheit, 2005), although identifiable glucose dehydrogenases and gluconate dehydratases (enzymes that lead to the formation of 2-keto-3-deoxygluconate) were lacking in our incomplete drafts. Interestingly, most drafts contained a putative glucose/sorbosone dehydrogenase (COG2133), but this enzyme has to our knowledge not been characterized in a member of the Halobacteria. Three of the Natrialbaceae-related drafts contained within the Natrialbaceae encoded a putative nonphosphorylative glyceraldehyde 3-phosphate dehydrogenase (gapN; EC 1.2.1.9), the other ten drafts contained one or both types of phosphorylative glyceraldehyde 3-phosphate dehydrogenase(s), namely GAPDH (EC 1.2.1.12) and/or gap2 (EC 1.2.1.59). The latter enzyme is part of the EmdenMeyerhoff pathway for hexose degradation, which has been shown in Halococcus saccharolyticus (family Halobacteriaceae) to be the main pathway for fructose degradation (Johnsen et al., 2001). The presence of a similarly branched EntnerDoudoroff/Emden-Meyerhof pathway is suspected to be the general case, since all drafts contained at least one of the enzymes typical for a modified Emden-Meyerhof pathway, which includes an ATP-dependent glucokinase, an ADP-dependent glucokinase/phosphofructokinase and a fructose-bisphosphate aldolase (both Class I and II found).

\section{Primary organic carbon degradation}

Polysaccharide-degrading potential was found in almost all Halobacteria-related draft genomes (Supplementary Table 11). Almost all drafts encoded glucoamylases and related glycoside hydrolases. Putative chitinases were encoded by Natrialbaceae archaeon B1-Br10_E2g27 and Haloferacaceae archaeon TcBr11_E2g18, with catalytic domains resembling those of GH18 and GH19 CAZy family proteins respectively. Putative cellulases were encoded by the Natrialbaceae archaea B1-Br10_E2g27, TcBr11_E2g14 and Tc-Br11_E2g28, and Haloferacaceae archaeon PL-Br10_E2g29. These particular organisms might be able to hydrolyze or even grow on recalcitrant organic substrates such as cellulose and chitin as shown previously for several Halobacteria isolated from soda lakes (Sorokin et al., 2015d). Some of these isolates remarkably grew both on celluloses and chitin. Interestingly, also draft genome B1-Br10_E2g27 held both cellulolytic and chitinolytic potential.

\section{Aerobic CO oxidation}

The draft genome of Natrialbaceae archaeon Tc-Br11_E2g1 contained coxM and coxS genes, the draft of Natrialbaceae archaeon Tc-Br11_E2g28 contained a coxL. Together these genes encode the three main subunits of a putative aerobic carbon monoxide dehydrogenase $(\mathrm{CODH})$. The large subunit (coxL) of $\mathrm{CODH}$ is a molybdopterin dehydrogenase FAD-binding protein with a $\mathrm{Mo}-\mathrm{Cu}$ sulfur-bridged cluster in its active site that catalyzes the oxidation of $\mathrm{CO}$ to $\mathrm{CO}_{2}$, yielding two electrons and two protons (Dobbek et al., 2001; Gnida et al., 2003; King and Weber, 2007). While the use of CO as a sole energy and carbon source or as an alternate energy source coupled to $\mathrm{CO}_{2}$ fixation ("carboxydotrophy") is typically restricted to few bacterial groups and can occur only under elevated CO concentrations, the use of $\mathrm{CO}$ at low concentrations as an alternative energy source ("carboxydovory") is suggested to be widespread amongst 
heterotrophs (King and Weber, 2007; Cunliffe, 2011; King, 2013). It was recently proposed that aerobic $\mathrm{CO}$ oxidation might have evolved in the phylum Euryarchaeota (genus Natronorubrum; King, 2013) and CO consumption has been demonstrated in isolates from the genus Halorubrum and Natronorubrum (King, 2015). Since, gammaproteobacterial carboxydotrophs as well as carboxydovores have been isolated from several soda lakes around the world (Sorokin et al., 2015a), CO must be to some extent available to soda lake aerobic microbes. As suggested previously for heterotrophic bacteria in the oceans (MartinCuadrado et al., 2009), Halobacteria might energetically benefit as well from metabolizing CO released by the photolysis of organic material in the surface waters of soda lakes. However, whether any members of the Euryarchaeota, including members of the Halobacteria, can actually use $\mathrm{CO}$ as an energy source remains to be proven.

\section{Bacteroidetes \\ Phylogeny}

Three Bacteroidetes-related draft genomes were obtained from Tc-Br11 and PL-Br10 (Table 3; Figure 2E; Supplementary Table 8). The draft genome T5-Br10_B2g13 was affiliated with the genera Gracilimonas and Balneola (order Sphingobacteriales; Chitinophagaceae, no rank; Urios et al., 2006; Choi et al., 2009), is only distantly related to the orders Flavobacteriales and Bacteroidetes Order II Incertae sedis and had an average GC content of $41 \%$. Similar phylogeny and GC content was reported for Gracilimonas and the related genus Balneola and annotated genes were affiliated consistently with these two genera (best-hit analysis). While Gracilimonas-related OTUs comprised up to $18.84 \%$ of total $16 \mathrm{~S}$ rRNA amplicon sequences from T5, $2.50 \%$ of Chitinophagaceae-related OTUs could not be assigned to a known genus (Table 2). Since, the ANIs of T5-Br10_B2g13 with the incomplete genomes available from Balneola vulgaris DSM 17893 and Gracilimonas tropica DSM 19535 were low (65.2 and $66.2 \%$ respectively), T5-Br10_B2g13 must belong to a novel genus within the Chitinophagaceae. Its closest cultured relative appears to be a proteolytic satellite (bacterial symbiont) of a soda lake filamentous cyanobacterium isolated from the same area (DY Sorokin, unpublished data). Notably in our genome tree, Gracilimonas, Balneola, and T5-Br10_B2g13 formed a separate clade from the other three genera within the family Chitinophagaceae (namely Chitinophaga, Niabella, and Niastella).

The draft genome T5-Br10_B2g0 was affiliated with the genus Psychroflexus (family Flavobacteriaceae; Bowman et al., 1998; Donachie et al., 2004; Yoon et al., 2009) in our genome tree. Since no Psychroflexus-related OTUs were found in the Flavobacteriaceae-related 16S rRNA amplicon sequences of T5 (Table 2) and ANIs of T5-Br10_B2g0 with the incomplete genomes available from Psychroflexus gondwanensis ACAM 44 and Psychroflexus tropicus DSM 15496 were low (68.4 and 68.2\% respectively), T5-Br10_B2g0 must represent the draft genome of a novel lineage within the Flavobacteriaceae.

The draft genome Tc-Br11-B2-g6_7 belonged to the family Rhodothermaceae (Bacteroidetes Order II Incertae sedis, currently order Cytophagales, class Cytophagia; Park et al., 2014) branching in a genome tree between the genera Rhodothermus and Salisaeta of which halophilic isolates have been obtained from marine hot springs and high salt environments respectively (Antón et al., 2002; Vaisman and Oren, 2009). Rhodothermaceae-related OTUs without genus assignment as well as Salisaeta-related OTUs were found in the $16 \mathrm{~S}$ rRNA amplicon sequences from Tc $(0.24$ and $0.06 \%$ of total OTUs respectively, Table 2 ). Since the ANI of Tc-Br-Bacteroidetes-g6_7 with the incomplete genome available from Salisaeta longa DSM 21114 was low (67.4\%), Tc-Br11-B2g6_7 belongs to a novel extreme halophilic Bacteroidetes lineage within the Rhodothermaceae.

\section{Central carbon metabolism and primary organic carbon degradation}

In all Bacteroidetes draft genomes cytochrome c oxidase subunits I and III, marker genes for aerobic respiration, were encoded (Supplementary Table 12), as well as a complete or a nearcomplete Embden-Meyerhof pathway, pyruvate dehydrogenase complex, and Krebs cycle. Genes encoding both subunits of the 2-oxoglutarate:ferredoxin oxidoreductase (EC 1.2.7.3) as well as a gene encoding a L-lactate dehydrogenase were found for Chitinophagaceae bacterium T5-Br10_B2g13 and Rhodothermaceae bacterium Tc-Br11_B2g6_7, suggesting they have the potential for sugar fermentation under anoxic or microoxic conditions.

Several putative glycoside hydrolases involved in polysaccharide degradation were encoded in the three Bacteroidetes draft genomes (Supplementary Table 13). Putative glucoamylases, cellulases, and related enzymes suggest amylolytic and cellulolytic potential for all three organisms. Hemicellulases and related enzymes were only found in Chitinophagaceae bacterium T5-Br10_B2g13 and Rhodothermaceae bacterium TcBr11_B2g6_7. Both Chitinophagaceae bacterium T5-Br10_B2g13 and Flavobacteriaceae bacterium T5-Br10_B2g0 possessed a putative $\beta$-galactosidase (lacZ) and functionally related glycoside hydrolases, as well as chitinase related enzymes (CAZy family GH 23).

\section{Denitrification: $\mathrm{N}_{2} \mathrm{O}$ reduction}

The genome of Chitinophagaceae bacterium T5-Br10_B2g13 encodes a key functional gene nos $Z$ for dissimilatory nitrous oxide $\left(\mathrm{N}_{2} \mathrm{O}\right)$ reduction within the denitrification pathway (Wood et al., 2001; Jones et al., 2013; Supplementary Table 12). According to the model of Geobacillus thermodenitrificans, the gene also lacked a characteristic Tat-signal at the N-terminus for translocation across the membrane (Liu et al., 2008). This finding agrees with the known widespread $\mathrm{N}_{2} \mathrm{O}$ reduction potential in various environments by members of the Firmicutes and Bacteroidetes which is encoded by a nos $Z$ with a presumed Sectranslocation pathway (Jones et al., 2013). Although it is known that some halophilic Halobacteria have the capacity to reduce $\mathrm{N}_{2} \mathrm{O}$ (Jones et al., 2013), the process was previously only shown for soda lake isolates within the Gammaproteobacteria belonging to the genera Halomonas, Thioalkalivibrio, and Alkalispirillum (Sorokin et al., 2012, 2014). Amongst the $>5 \mathrm{~kb}$ contigs we found nos $Z$ genes in the three most saline datasets related to the genera Halorubrum and Natronococcus, but we did not find gammaproteobacterial nos $Z$ genes in the $>5 \mathrm{~kb}$ contigs of T5. 


\section{Osmotic adaptation: rhodopsins, salt-in strategy and organic osmolytes}

Finally, both Chitinophagaceae bacterium T5-Br10_B2g13 and Flavobacteriaceae bacterium T5-Br10_B2g0 contained a putative sodium pumping rhodopsin with characteristic NQ motif, which could aid in keeping the intracellular sodium ion concentration low (Inoue et al., 2013; Kwon et al., 2013; Supplementary Table 9). Flavobacteriaceae bacterium T5-Br10_B2g0 possesses a putative proton pumping proteorhodopsin as well (Béja et al., 2001). Maintaining osmotic balance with high levels of cytoplasmic $\mathrm{KCl}$, often referred to as a "salt-in strategy" (Oren, 2013), appears to be a common strategy for members of the Halobacteria and Nanohaloarchaea thriving in hypersaline habitats (Ghai et al., 2011; Youssef et al., 2014; Figure 3; Supplementary Figure 3). Chitinophagaceae bacterium T5Br10_B2g13 and Rhodothermaceae bacterium Tc-Br11_B2g6_7 seem to deploy this strategy to some extent since their predicted proteomes were enriched in acidic residues compare to that of E. coli, despite the fact that putative genes for the synthesis and uptake of organic osmolytes (including betaine aldehyde dehydrogenases and betaine/carnitine/choline transporters) were found in their draft genomes. So far, the "salt-in strategy" was shown only for two bacterial phyla, in the orders Halanaerobiales and Natranaerobiales (Firmicutes; Oren, 2013) and Salinibacter (Bacteroidetes; Mongodin et al., 2005). No acid-shifted proteome compared to E. coli was found for Flavobacteriaceae bacterium T5-Br10_B2g0.

\section{Natural Abundance of the Reconstructed Genomes}

In agreement with the $16 \mathrm{~S}$ amplicon sequencing, Haloferacaceaerelated genomes were the most abundant in the three most saline brines of B1, Tc and PL (Figure 4). Although the putative chitinolytic Haloferacaceae archaeon Tc-Br11_E2g18 was by far the most abundant organism in the brine of $\mathrm{B} 1$, the most saline brine still harbored a considerable diverse population of other Halobacteria (Figure 4A). The dominant high-GC Halobacteria in the most saline brines left their signature in the GC profiles of the raw metagenomic reads (Supplementary Figure 2). Nanohaloarchaea-related genomes were confined to the most saline brines and were moderately (reads per kilobase of sequenced reads per gigabase of mapped reads (RPKG) between 120 and 20, Figure 4B) or low abundant (RPKG between 20 and 2, Figure 4C). Previous nanohaloarchaeal metagenomic genome reconstructions were only obtained from more moderate salinities below $250 \mathrm{~g} / \mathrm{L}$ of total salts (Ghai et al., 2011; Narasingarao et al., 2012; Martínez-García et al., 2014). Only one of the Halobacteria-related genomes, Haloferacaceae archaeon PL-Br10_E2g46, was moderately abundant in the brine of T5 with a salinity of $170 \mathrm{~g} / \mathrm{L}$. It was even more abundant than the Bacteroidetes-related genomes that were confined to the brine of T5 (using an arbitrary cut-off of $\mathrm{RPKG}=2$ for presence of a certain organism within a dataset) and Thioalkalivibrio sp. AKL9 (class Gammaproteobacteria). Rhodothermaceae bacterium Tc-Br11_B2g6_7 had only a low abundance in the brine of Tc. The draft genomes that were reconstructed from a different brine, but were identified to be the same species, Natrialbaceae archaeon PL-Br10_E2g26 \&
Tc-Br11_E2g28 and Nanohaloarchaea archaeon B1-Br10_U2g1 and PL-Br10_U2g19, showed comparable RPKG for all four datasets. Finally, only very low read abundance of our soda lake draft genomes was found in the metagenomics datasets SP-37 and SP-19, originating from salterns with neutral $\mathrm{pH}$. Although Halobacteria, Nanohaloarchaea, and Bacteroidetes are common taxa in high-salt environments, the specific organisms belonging to these groups that can thrive at high alkalinities are likely to differ from those that thrive at circum-neutral $\mathrm{pH}$. The same holds true for pure cultures, where the isolates from soda lakes in most cases differentiate from their halophilic counterparts on the genus level (Sorokin et al., 2014, 2015a).

\section{CONCLUSIONS AND FUTURE RESEARCH}

Salinity has a similar dominant influence on the overall prokaryote community structure of alkaline soda lake brines as it has on that of hypersaline ponds of solar salterns with neutral $\mathrm{pH}$; over a salt concentration of roughly $250 \mathrm{~g} / \mathrm{L}$ bacterial abundance declines and dominance shifts to members of the Halobacteria (Rodriguez-Valera et al., 1985; Oren, 1994; Ghai et al., 2011). Yet hypersaline soda lake brines with salinities exceeding 250 $\mathrm{g} / \mathrm{L}$ harbor considerably different and more diverse communities compared to solar saltern brines of similar salinities. The absence of calcium salts that precipitate at high $\mathrm{pH}$ and much lower prevalent magnesium concentrations could offer explanations for the differences found in the community structure. Furthermore, the investigated, shallow soda lakes from the Kulunda Steppe have a very unstable water regime with large fluctuations in salinity throughout the seasons and the year, according to local snow melt, groundwater inflow and evaporation rates. These fluctuations could also contribute to sustaining such diverse soda brine communities. Finally, the nearly $50 \%$ lower osmotic pressure in hypersaline soda lake brines compared to solar salterns (Banciu and Sorokin, 2013; Sorokin et al., 2015a) might be the reason why in particular Nanohaloarchaea, which rely solely on the salt-in strategy for osmotic adaption, prefer higher salinities in alkaline brines than in $\mathrm{NaCl}$ brines with circumneutral $\mathrm{pH}$.

By reconstructing the first draft genomes from members of the candidate class Nanohaloarchaea present in hypersaline soda brines, we extend the amount of available genomic data from this enigmatic new group of Archaea considerably and suggest that their inferred ecological lifestyle with respect to oxygen respiration should be revised. Furthermore, we identified hydrolytic capacity not only within previously unrecognized members of the Bacteroidetes, but also amongst members of the Halobacteria which outnumber the first in the most saline brines. The fact that some members of the Halobacteria might fulfill a primary rather than a secondary degrading function in the organic carbon cycle of soda lakes is supported by previous culture-dependent studies. Overall, the dominant organisms in the most saline brines seem to be aerobic heterotrophs that adapted to the presence of light and to the low solubility of oxygen at high salinities with putative sugar fermentative capabilities. We identified two members of the Bacteroidetes that might utilize light not simply as an additional energy source or 


\section{A}

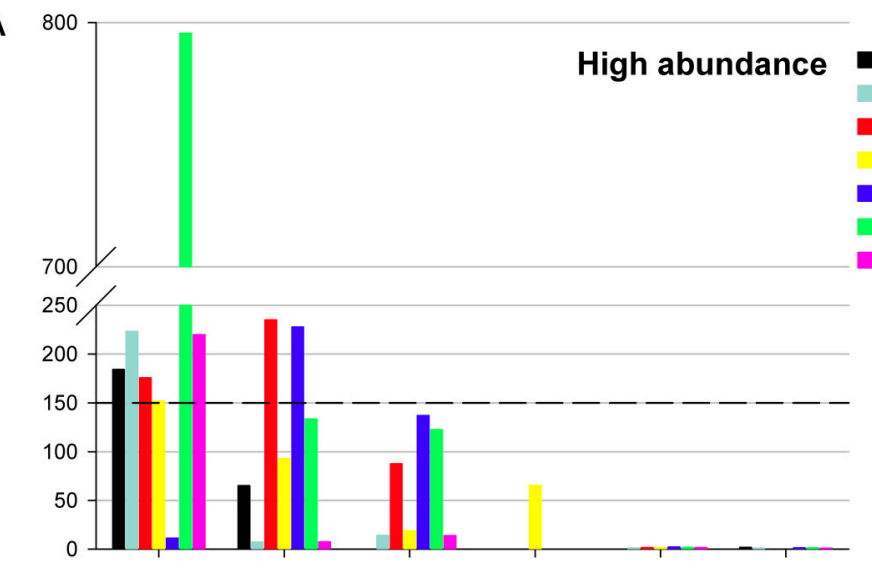

B

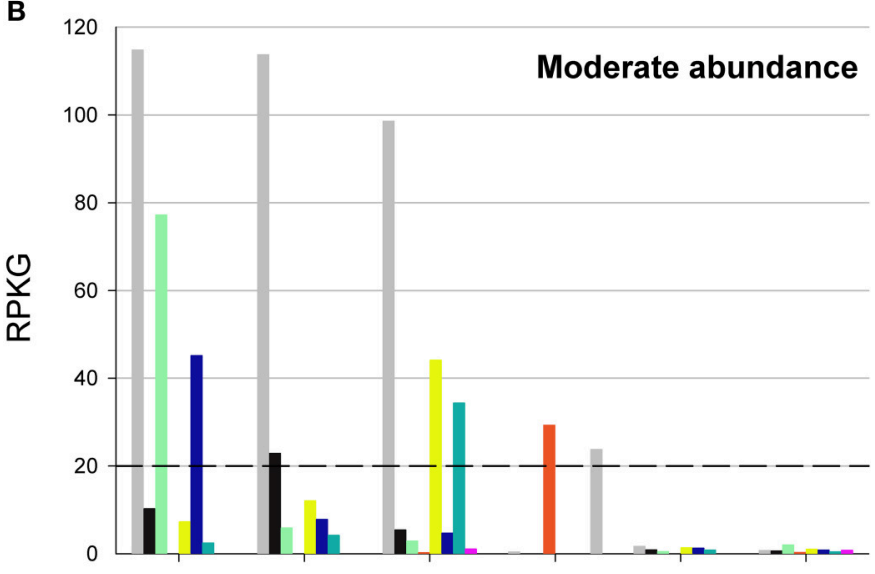

C

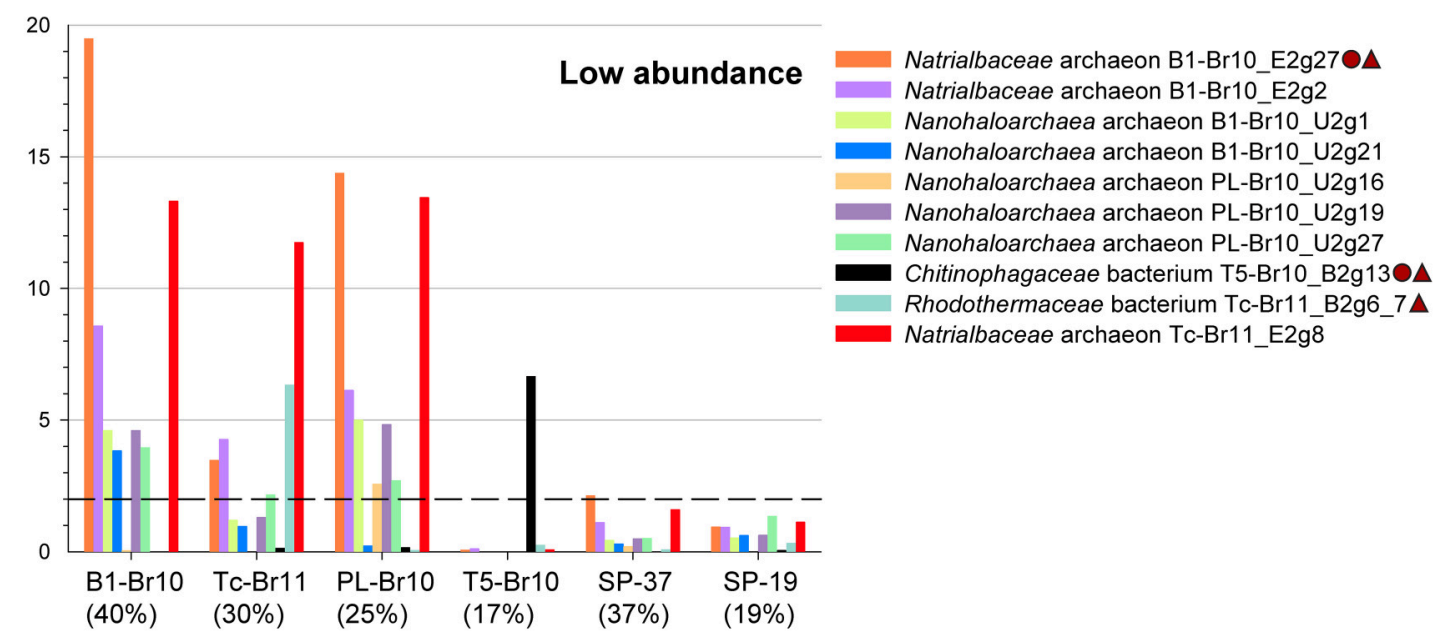

FIGURE 4 | Read recruitments (<95\% N-identity) of reconstructed draft genomes and a reference draft genome from the genus Thioalkalivibrio from different metagenomic datasets. SP-37 and SP-19 are metagenomes from a crystallizer and an intermediate saline pond of the solar saltern in Santa Pola, Spain, with 37 and 19\%, salinity respectively (Ghai et al., 2011). The salinity (in \%) for the different soda brine metagenomes is given between brackets. Reconstructed draft genomes encoding putative chitinases are marked in the figure legend with a circle, those encoding putative cellulases with a triangle. RPKG, Reads Per Kilobase of sequenced reads per Gigabase of mapped reads. (A) Putative organisms with relatively high abundance (> 150 RPKG), (B) with moderate abundance, (C) and with relatively low abundance $(<20 \mathrm{RPKG})$.

sensory input for movement, but rather to pump out $\mathrm{Na}^{+}$via sodium pumping rhodopsins.

The presented genomic data provide a first insight into the physiological potential of a wealth of still uncultured organisms living in hypersaline soda lake brines. We hope this study can pave the way for the design of novel isolation strategies and protein structure-function studies, crucial in obtaining unequivocal evidence for the proposed metabolic 
features of the novel organisms. More in depth sampling over time or space of soda brines could bring a more detailed understanding of the influence of environmental gradients in salinity and salt composition, as well as of other parameters such as $\mathrm{pH}$ and seasonality, on specific microbial populations and functional groups. Future research should combine transcriptomic and/or proteomic approaches with in situ rates of element transformations to reveal the active prokaryote community members in hypersaline soda lake brines in relation to salinity and could aim at verifying the functional role and relative importance of rare vs. abundant community members.

\section{AUTHOR CONTRIBUTIONS}

DS, $\mathrm{PH}$, and GM were responsible for the conception of the work. DS collected the samples and extracted the DNA. GM prepared samples for amplicon sequencing and performed subsequent analysis. ST was responsible for the DNA sequencing. RG performed the assembly of the metagenomic reads and assisted $\mathrm{CV}$ with the genome reconstructions and subsequent analysis. $\mathrm{CV}$ integrated and interpreted all data and is the primary author of the manuscript. She received thereby substantial contributions

\section{REFERENCES}

Albertsen, M., Hugenholtz, P., Skarshewski, A., Nielsen, K. L., Tyson, G. W., and Nielsen, P. H. (2013). Genome sequences of rare, uncultured bacteria obtained by differential coverage binning of multiple metagenomes. Nat. Biotechnol. 31, 533-538. doi: 10.1038/nbt.2579

Alneberg, J., Bjarnason, B. S., de Bruijn, I., Schirmer, M., Quick, J., Ijaz, U. Z., et al. (2014). Binning metagenomic contigs by coverage and composition. Nat. Methods 11, 1144-1146. doi: 10.1038/nmeth.3103

Andrade, K., Logemann, J., Heidelberg, K. B., Emerson, J. B., Comolli, L. R., Hug, L. A., et al. (2015). Metagenomic and lipid analyses reveal a diel cycle in a hypersaline microbial ecosystem. ISME J. 9, 2697-2711. doi: 10.1038/ismej.2015.66

Antón, J., Oren, A., Benlloch, S., Rodríguez-Valera, F., Amann, R., and RossellóMora, R. (2002). Salinibacter ruber gen. nov., sp. nov., a novel, extremely halophilic member of the bacteria from saltern crystallizer ponds. Int. J. Syst. Evol. Microbiol. 52, 485-491. doi: 10.1099/00207713-52-2-485

Antón, J., Rosselló-Mora, R., Rodríguez-Valera, F., and Amann, R. (2000). Extremely halophilic bacteria in crystallizer ponds from solar salterns. Appl. Environ. Microbiol. 66, 3052-3057. doi: 10.1128/AEM.66.7.3052-3057.2000

Asao, M., Pinkart, H. C., and Madigan, M. T. (2011). Diversity of extremophilic purple phototrophic bacteria in soap lake, a central washington (USA) soda lake. Environ. Microbiol. 13, 2146-2157. doi: 10.1111/j.1462-2920.2011. 02449.x

Ausec, L., Zakrzewski, M., Goesmann, A., Schlüter, A., and Mandic-Mulec, I. (2011). Bioinformatic analysis reveals high diversity of bacterial genes for laccase-like enzymes. PLoS ONE 6:e25724. doi: 10.1371/journal.pone.0025724

Banciu, H. L., and Sorokin, D. Y. (2013). "Adaptation in Haloalkaliphiles and Natronophilic bacteria," in Polyextremophiles, eds J. Seckbach, A. Oren, and H. Stan-Lotter (Dordrecht: Springer), 121-178.

Bateman, A., Coin, L., Durbin, R., Finn, R. D., Hollich, V., Griffiths-Jones, S., et al. (2004). The Pfam protein families database. Nucleic Acids Res. 32(Suppl. 1), D138-D141. doi: 10.1093/nar/gkh121

Bauer, M., Kube, M., Teeling, H., Richter, M., Lombardot, T., Allers, E., et al. (2006). Whole genome analysis of the marine Bacteroidetes 'Gramella forsetii' reveals adaptations to degradation of polymeric organic matter. Environ. Microbiol. 8, 2201-2213. doi: 10.1111/j.1462-2920.2006.01152.x of all co-authors, including final approval for this version to be published.

\section{FUNDING}

CV and GM are supported by the ERC Advanced Grant PARASOL (No. 322551). The work of CV was further supported by an STSM Grant from the COST Action ES1103. RG is partially supported by the Grant Agency of the Czech Science Foundation under the research grant 13-00243S. FR-V and RG are supported by a grant from the European Union (MaCuMBA project, grant 311975). DS is supported by the Russian Foundation for Basic Research (16-04-00035). The work conducted by the U.S. Department of Energy Joint Genome Institute, a DOE Office of Science User Facility, is supported under Contract No. DE-AC02$05 \mathrm{CH} 11231$.

\section{SUPPLEMENTARY MATERIAL}

The Supplementary Material for this article can be found online at: http://journal.frontiersin.org/article/10.3389/fmicb. 2016.00211
Béja, O., Spudich, E. N., Spudich, J. L., Leclerc, M., and DeLong, E. F. (2001). Proteorhodopsin phototrophy in the ocean. Nature 411, 786-789. doi: $10.1038 / 35081051$

Benlloch, S., López-López, A., Casamayor, E. O., Øvreås, L., Goddard, V., Daae, F. L., et al. (2002). Prokaryotic genetic diversity throughout the salinity gradient of a coastal solar saltern. Environ. Microbiol. 4, 349-360. doi: 10.1046/j.14622920.2002.00306.x

Boldareva, E. N., Akimov, V. N., Boychenko, V. A., Stadnichuk, I. N., Moskalenko, A. A., Makhneva, Z. K., et al. (2008). Rhodobaca barguzinensis sp. nov., a new alkaliphilic purple nonsulfur bacterium isolated from a soda lake of the Barguzin Valley (Buryat Republic, Eastern Siberia). Microbiology 77, 206-218. doi: 10.1134/S0026261708020148

Boldareva, E. N., Bryantseva, I. A., Tsapin, A., Nelson, K., Sorokin, D. Y., Tourova, T. P., et al. (2007). The new alkaliphilic bacteriochlorophyll a-containing bacterium Roseinatronobacter monicus sp. nov. from the hypersaline Soda Mono Lake (California, United States). Microbiology 76, 82-92. doi: 10.1134/ S0026261707010122

Bowman, J. P., McCammon, S. A., Lewis, T., Skerratt, J. H., Brown, J. L., Nichols, D. S., et al. (1998). Psychroflexus torquis gen. nov., sp. nov. a psychrophilic species from Antarctic sea ice, and reclassification of Flavobacterium gondwanense (Dobson et al., 1993) as Psychroflexus gondwanense gen. nov., comb. nov. Microbiology 144, 1601-1609. doi: 10.1099/00221287-1446-1601

Cabello, P., Roldán, M. D., and Moreno-Vivián, C. (2004). Nitrate reduction and the nitrogen cycle in Archaea. Microbiology 150, 3527-3546. doi: 10.1099/mic.0.27303-0

Casamayor, E. O., Calderón-Paz, J. I., and Pedrós-Alió, C. (2000). 5S rRNA fingerprints of marine Bacteria, halophilic Archaea and natural prokaryotic assemblages along a salinity gradient. FEMS Microbiol. Ecol. 34, 113-119. doi: 10.1111/j.1574-6941.2000.tb00760.x

Casamayor, E. O., Massana, R., Benlloch, S., Øvreås, L., Díez, B., Goddard, V. J., et al. (2002). Changes in archaeal, bacterial and eukaryal assemblages along a salinity gradient by comparison of genetic fingerprinting methods in a multipond solar saltern. Environ. Microbiol. 4, 338-348. doi: 10.1046/j.14622920.2002.00297.x

Castelle, C. J., Wrighton, K. C., Thomas, B. C., Hug, L. A., Brown, C. T., Wilkins, M. J., et al. (2015). Genomic expansion of domain archaea highlights roles for 
organisms from new phyla in anaerobic carbon cycling. Curr. Biol. 25, 690-701. doi: 10.1016/j.cub.2015.01.014

Cho, Y., Chung, H., Jang, G. I., Choi, D. H., Noh, J. H., and Cho, B. C. (2013). Gracilimonas rosea sp. nov., isolated from tropical seawater, and emended description of the genus Gracilimonas. Int. J. Syst. Evol. Microbiol. 63, 4006-4011. doi: 10.1099/ijs.0.052340-0

Choi, D. H., Zhang, G. I., Noh, J. H., Kim, W. S., and Cho, B. C. (2009). Gracilimonas tropica gen. nov., sp. nov., isolated from a Synechococcus culture. Int. J. Syst. Evol. Microbiol. 59, 1167-1172. doi: 10.1099/ijs.0.005512-0

Cole, J. R., Wang, Q., Fish, J. A., Chai, B., McGarrell, D. M., Sun, Y., et al. (2014). Ribosomal database project: data and tools for high throughput rRNA analysis. Nucleic Acids Res. 41(Database issue):gkt1244. doi: 10.1093/nar/gkt1244

Cox, M. P., Peterson, D. A., and Biggs, P. J. (2010). SolexaQA: at-a-glance quality assessment of Illumina second-generation sequencing data. $B M C$ Bioinformatics 11:485. doi: 10.1186/1471-2105-11-485

Cunliffe, M. (2011). Correlating carbon monoxide oxidation with cox genes in the abundant marine Roseobacter clade. ISME J. 5, 685-691. doi: 10.1038/ismej.2010.170

De Castro, R. E., Maupin-Furlow, J. A., Giménez, M. I., Seitz, M. K. H., and Sánchez, J. J. (2006). Haloarchaeal proteases and proteolytic systems. FEMS Microbiol. Rev. 30, 17-35. doi: 10.1111/j.1574-6976.2005.00003.x

Dillon, J. G., Carlin, M., Gutierrez, A., Nguyen, V., and McLain, N. (2013). Patterns of microbial diversity along a salinity gradient in the Guerrero Negro solar saltern, Baja CA Sur, Mexico. Front. Microbiol. 4:399. doi: 10.3389/fmicb.2013.00399

Dimitriu, P. A., Pinkart, H. C., Peyton, B. M., and Mormile, M. R. (2008). Spatial and temporal patterns in the microbial diversity of a meromictic soda lake in washington state. Appl. Environ. Microbiol. 74, 4877-4888. doi: 10.1128/AEM.00455-08

Dobbek, H., Svetlitchnyi, V., Gremer, L., Huber, R., and Meyer, O. (2001). Crystal structure of a carbon monoxide dehydrogenase reveals a [Ni-4Fe-5S] cluster. Science 293, 1281-128510. doi: 10.1126/science. 1061500

Donachie, S. P., Bowman, J. P., and Alam, M. (2004). Psychroflexus tropicus sp. nov., an obligately halophilic Cytophaga-Flavobacterium-Bacteroides group bacterium from an Hawaiian hypersaline lake. Int. J. Syst. Evol. Microbiol. 54, 935-940. doi: 10.1099/ijs.0.02733-0

Fernández, A. B., Ghai, R., Martin-Cuadrado, A., Sánchez-Porro, C., Rodriguez-Valera, F., and Ventosa, A. (2014a). Prokaryotic taxonomic and metabolic diversity of an intermediate salinity hypersaline habitat assessed by metagenomics. FEMS Microbiol. Ecol. 88, 623-635. doi: 10.1111/1574-6941.12329

Fernández, A. B., Vera-Gargallo, B., Sánchez-Porro, C., Ghai, R., Papke, R. T., Rodriguez-Valera, F., et al. (2014b). Comparison of prokaryotic community structure from Mediterranean and Atlantic saltern concentrator ponds by a metagenomic approach. Front. Microbiol. 5:196. doi: 10.3389/fmicb.2014.00196

Foti, M. J., Sorokin, D. Y., Zacharova, E. E., Pimenov, N. V., Kuenen, J. G., and Muyzer, G. (2008). Bacterial diversity and activity along a salinity gradient in soda lakes of the Kulunda Steppe (Altai, Russia). Extremophiles 12, 133-145. doi: 10.1007/s00792-007-0117-7

Gareeb, A. P., and Setati, M. E., (2009). Assessment of alkaliphilic haloarchaeal diversity in Sua pan evaporator ponds in Botswana. Afr. J. Biotechnol. 8, 259-267. doi: 10.5897/AJB2009.000-9046

Ghai, R., Pašić, L., Fernández, A. B., Martin-Cuadrado, A., Mizuno, C. M., McMahon, K. D., et al. (2011). New abundant microbial groups in aquatic hypersaline environments. Sci. Rep. 1:135. doi: 10.1038/srep00135

Gnida, M., Ferner, R., Gremer, L., Meyer, O., and Meyer-Klaucke, W. (2003). A novel binuclear [CuSMo] cluster at the active site of carbon monoxide dehydrogenase: characterization by $\mathrm{X}$-ray absorption spectroscopy. Biochemistry 42, 222-230. doi: 10.1021/bi026514n

Goris, J., Konstantinidis, K. T., Klappenbach, J. A., Coenye, T., Vandamme, P., and Tiedje, J. M. (2007). DNA-DNA hybridization values and their relationship to whole-genome sequence similarities. Int. J. Syst. Evol. Microbiol. 57, 81-91. doi: 10.1099/ijs.0.64483-0

Grant, S., Grant, W. D., Jones, B. E., Kato, C., and Li, L. (1999). Novel archaeal phylotypes from an East African alkaline saltern. Extremophiles 3, 139-145. doi: 10.1007/s007920050109

Grant, W. D. (2004). Life at low water activity. Philos. Trans. R. Soc. B Biol. Sci. 359, 1249-1267. doi: 10.1098/rstb.2004.1502
Gupta, R. S., Naushad, S., and Baker, S. (2014). Phylogenomic analyses and molecular signatures for the class Halobacteria and its two major clades: a proposal for division of the class Halobacteria into an emended order Halobacteriales and two new orders, Haloferacales ord. nov. and Natrialbales ord. nov. Int. J. Syst. Evol. Microbiol. 65, 1050-1069. doi: 10.1099/ijs.0.070136-0

Haft, D. H., Loftus, B. J., Richardson, D. L., Yang, F., Eisen, J. A., Paulsen, I. T., et al. (2001). TIGRFAMs: a protein family resource for the functional identification of proteins. Nucleic Acids Res. 29, 41-43. doi: 10.1093/nar/29.1.41

Hagedoorn, P. L., Chen, T., Schröder, I., Piersma, S. R., de Vries, S., and Hagen, W. R. (2005). Purification and characterization of the tungsten enzyme aldehyde: ferredoxin oxidoreductase from the hyperthermophilic denitrifier Pyrobaculum aerophilum. J. Biol. Inorg. Chem. 10, 259-269. doi: 10.1007/s00775-005-0637-5

Huang, Y., Gilna, P., and Li, W. (2009). Identification of ribosomal RNA genes in metagenomic fragments. Bioinformatics 25, 1338-1340. doi: 10.1093/bioinformatics/btp161

Hügler, M., Huber, H., Molyneaux, S. J., Vetriani, C., and Sievert, S. M. (2007). Autotrophic $\mathrm{CO}_{2}$ fixation via the reductive tricarboxylic acid cycle in different lineages within the phylum Aquificae: evidence for two ways of citrate cleavage. Environ. Microbiol. 9, 81-92. doi: 10.1111/j.1462-2920.2006.01118.x

Humayoun, S. B., Bano, N., and Hollibaugh, J. T. (2003). Depth distribution of microbial diversity in mono lake, a meromictic soda lake in California. Appl. Environ. Microbiol. 69, 1030-1042. doi: 10.1128/AEM.69.2.1030-10 42.2003

Hyatt, D., Chen, G. L., LoCascio, P. F., Land, M. L., Larimer, F. W., and Hauser, L. J. (2010). Prodigal: prokaryotic gene recognition and translation initiation site identification. BMC Bioinformatics 11:119. doi: 10.1186/1471-2105-11-119

Imhoff, J. F. (1995). "Taxonomy and physiology of phototrophic purple bacteria and green sulfur bacteria," in Anoxygenic Photosynthetic Bacteria, eds R. E. Blankenship, M. T. Madigan and C. E. Bauer (Dordrecht: Springer), 1-15.

Inoue, K., Ono, H., Abe-Yoshizumi, R., Yoshizawa, S., Ito, H., Kogure, K., et al. (2013). A light-driven sodium ion pump in marine bacteria. Nat. Commun. 4:1678. doi: 10.1038/ncomms 2689

Isachenko, B. L. (1951). "Chloride, sulfate and soda lakes of the Kulunda steppe and the biogenic process in them," in Selected Works, Vol. 2 (Leningrad: Academy of Sciences USSR), 143-162.

Johnsen, U., Selig, M., Xavier, K. B., Santos, H., and Schönheit, P. (2001). Different glycolytic pathways for glucose and fructose in the halophilic archaeon Halococcus saccharolyticus. Arch. Microbiol. 175, 52-61. doi: $10.1007 / \mathrm{s} 002030000237$

Jones, B. F., Eugster, H. P., and Rettig, S. L. (1977). Hydrochemistry of the Lake Magadi basin, Kenya. Geochim. Cosmochim. Acta 41, 53-72. doi: 10.1016/00167037(77)90186-7

Jones, C. M., Graf, D. R., Bru, D., Philippot, L., and Hallin, S. (2013). The unaccounted yet abundant nitrous oxide-reducing microbial community: a potential nitrous oxide sink. ISME J. 7, 417-426. doi: 10.1038/ismej.2012.125

Jormakka, M., Yokoyama, K., Yano, T., Tamakoshi, M., Akimoto, S., Shimamura, T., et al. (2008). Molecular mechanism of energy conservation in polysulfide respiration. Nat. Struct. Mol. Biol. 15, 730-737. doi: 10.1038/nsmb.1434

Keshri, J., Mody, K., and Jha, B. (2013). Bacterial community structure in a semiarid haloalkaline soil using culture independent method. Geomicrobiol. J. 30, 517-529. doi: 10.1080/01490451.2012.737092

King, C. E. (2013). Diversity and Activity of Aerobic Thermophilic Carbon Monoxide-Oxidizing Bacteria on Kilauea Volcano, Hawaii. Ph.D. thesis, Louisiana State University.

King, G. M. (2015). Carbon monoxide as a metabolic energy source for extremely halophilic microbes: implications for microbial activity in Mars regolith. Proc. Natl. Acad. Sci. U.S.A. 112, 4465-4470. doi: 10.1073/pnas.1424989112

King, G. M., and Weber, C. F. (2007). Distribution, diversity and ecology of aerobic CO-oxidizing bacteria. Nat. Rev. Microbiol. 5, 107-118. doi: 10.1038/nrmicrol595

Kompantseva, E. I., Bryantseva, I. A., Komova, A. V., and Namsaraev, B. B. (2007). The structure of phototrophic communities of soda lakes of the southeastern Transbaikal Region. Microbiology 76, 211-219. doi: 10.1134/S0026261707020130

Kompantseva, E. I., Komova, A. V., and Sorokin, D. Y. (2010). Communities of anoxygenic phototrophic bacteria in the saline soda lakes of the Kulunda Steppe (Altai region). Microbiology 79, 89-95. doi: 10.1134/S0026261710010121 
Kwon, S. K., Kim, B. K., Song, J. Y., Kwak, M. J., Lee, C. H., Yoon, J. H., et al. (2013). Genomic makeup of the marine flavobacterium Nonlabens (Donghaeana) dokdonensis and identification of a novel class of rhodopsins. Genome Biol. Evol. 5, 187-199. doi: 10.1093/gbe/evs134

Lanzen, A., Simachew, A., Gessesse, A., Chmolowska, D., Jonassen, I., and Øvreås, L. (2013). Surprising prokaryotic and eukaryotic diversity, community structure and biogeography of Ethiopian soda lakes. PLoS ONE 8:e72577. doi: 10.1371/journal.pone.0072577

Lassmann, T., and Sonnhammer, E. L. (2005). Kalign-an accurate and fast multiple sequence alignment algorithm. BMC Bioinformatics 6:298. doi: 10.1186/14712105-6-298

Lauro, F. M., DeMaere, M. Z., Yau, S., Brown, M. V., Ng, C., Wilkins, D., et al. (2011). An integrative study of a meromictic lake ecosystem in Antarctica. ISME J. 5, 879-895. doi: 10.1038/ismej.2010.185

León, M. J., Fernández, A. B., Ghai, R., Sánchez-Porro, C., Rodriguez-Valera, F., and Ventosa, A., (2014). From metagenomics to pure culture: isolation and characterization of the moderately halophilic bacterium Spiribacter salinus gen. nov., sp. nov. Appl. Environ. Microbiol. 80, 3850-3857. doi: 10.1128/AEM.00430-14

Li, D., Liu, C. M., Luo, R., Sadakane, K., and Lam, T. W. (2015). MEGAHIT: an ultra-fast single-node solution for large and complex metagenomics assembly via succinct de Bruijn graph. Bioinformatics 31, 1674-1676. doi: 10.1093/bioinformatics/btv033

Liu, X., Gao, C., Zhang, A., Jin, P., Wang, L., and Feng, L. (2008). The nos gene cluster from gram-positive bacterium Geobacillus thermodenitrificans NG802 and functional characterization of the recombinant NosZ. FEMS Microbiol. Lett. 289, 46-52. doi: 10.1111/j.1574-6968.2008.01362.x

Llorens-Marès, T., Yooseph, S., Goll, J., Hoffman, J., Vila-Costa, M., Borrego, C. M., et al. (2015). Connecting biodiversity and potential functional role in modern euxinic environments by microbial metagenomics. ISME J. 9, 1648-1661. doi: 10.1038/ismej.2014.254

Lombard, V., Golaconda Ramulu, H., Drula, E., Coutinho, P. M., and Henrissat, B. (2014). The carbohydrate-active enzymes database (CAZy) in 2013. Nucleic Acids Res. 42(Database issue):D490-D495. doi: 10.1093/nar/gkt1178

López-Pérez, M., Ghai, R., Leon, M. J., Rodríguez-Olmos, Á., Copa-Patiño, J. L., Soliveri, J., et al. (2013). Genomes of "Spiribacter," a streamlined, successful halophilic bacterium. BMC Genomics 14:787. doi: 10.1186/1471-2164-14-787

Lowe, T. M., and Eddy, S. R. (1997). tRNAscan-SE: a program for improved detection of transfer RNA genes in genomic sequence. Nucleic Acids Res. 25, 955-964. doi: 10.1093/nar/25.5.0955

Lynch, M. D., and Neufeld, J. D. (2015). Ecology and exploration of the rare biosphere. Nat. Rev. Microbiol. 13, 217-229. doi: 10.1038/nrmicro3400

Marmur, J. (1961). A procedure for isolation of DNA from microorganisms. J. Mol. Biol. 3, 208-214. doi: 10.1016/S0022-2836(61)80047-8

Martin-Cuadrado, A. B., Ghai, R., Gonzaga, A., and Rodriguez-Valera, F. (2009). CO dehydrogenase genes found in metagenomic fosmid clones from the deep Mediterranean Sea. Appl. Environ. Microbiol. 75, 7436-7444. doi: 10.1128/AEM.01283-09

Martin-Cuadrado, A. B., Pašiæ, L., and Rodriguez-Valera, F. (2015). Diversity of the cell-wall associated genomic island of the archaeon Haloquadratum walsbyi. BMC Genomics 16:603. doi: 10.1186/s12864-015-1794-8

Martínez-García, M., Santos, F., Moreno-Paz, M., Parro, V., and Antón, J. (2014). Unveiling viral-host interactions within the 'microbial dark matter'. Nat. Commun. 5:4542. doi: 10.1038/ncomms5542

Melack, J. M. (1981). Photosynthetic activity of phytoplankton in tropical African soda lakes. Hydrobiologia 81, 71-85. doi: 10.1007/BF00048707

Mesbah, N. M., Abou-El-Ela, S. H., and Wiegel, J. (2007). Novel and unexpected prokaryotic diversity in water and sediments of the alkaline, hypersaline lakes of the Wadi An Natrun, Egypt. Microbiol. Ecol. 54, 598-617. doi: 10.1007/s00248006-9193-y

Milford, A. D., Achenbach, L. A., Jung, D. O., and Madigan, M. T. (2000). Rhodobaca bogoriensis gen. nov. and sp. nov., an alkaliphilic purple nonsulfur bacterium from African Rift Valley soda lakes. Arch. Microbiol. 174, 18-27. doi: 10.1007/s002030000166

Mongodin, E. F., Nelson, K. E., Daugherty, S., Deboy, R. T., Wister, J., Khouri, H., et al. (2005). The genome of Salinibacter ruber: convergence and gene exchange among hyperhalophilic Bacteria and Archaea. Proc. Natl. Acad. Sci. U.S.A. 102, 18147-18152. doi: 10.1073/pnas.0509073102
Moriya, Y., Itoh, M., Okuda, S., Yoshizawa, A., and Kanehisa, M. (2007). KAAS: an automatic genome annotation and pathway reconstruction server. Nucleic Acids Res. 35, 182-185. doi: 10.1093/nar/gkm321

Mwirichia, R., Cousin, S., Muigai, A. W., Boga, H. I., and Stackebrandt, E. (2011) Bacterial diversity in the haloalkaline Lake Elmenteita, Kenya. Curr. Microbiol. 62, 209-221. doi: 10.1007/s00284-010-9692-4

Narasingarao, P., Podell, S., Ugalde, J. A., Brochier-Armanet, C., Emerson, J. B., Brocks, J. J., et al. (2012). De novo metagenomic assembly reveals abundant novel major lineage of Archaea in hypersaline microbial communities. ISME J. 6, 81-93. doi: 10.1038/ismej.2011.78

Ochsenreiter, T., Pfeifer, F., and Schleper, C. (2002). Diversity of Archaea in hypersaline environments characterized by molecular-phylogenetic and cultivation studies. Extremophiles 6, 267-274. doi: 10.1007/s00792-001-0253-4

Oren, A. (1994). The ecology of the extremely halophilic Archaea. FEMS Microbiol. Rev. 13, 415-439. doi: 10.1111/j.1574-6976.1994.tb00060.x

Oren, A. (2013). Life at high salt concentrations, intracellular $\mathrm{KCl}$ concentrations, and acidic proteomes. Front. Microbiol. 4:315. doi: 10.3389/fmicb.2013.00315

Oren, A. (2014). Taxonomy of halophilic Archaea: current status and future challenges. Extremophiles 18, 825-834. doi: 10.1007/s00792-014-0654-9

Pagaling, E., Wang, H., Venables, M., Wallace, A., Grant, W. D., Cowan, D. A., et al. (2009). Microbial biogeography of six salt lakes in inner Mongolia, China, and a salt lake in Argentina. Appl. Environ. Microbiol. 75, 5750-5760. doi: 10.1128/AEM.00040-09

Park, B. H., Karpinets, T. V., Syed, M. H., Leuze, M. R., and Uberbacher, E. C. (2010). CAZymes Analysis Toolkit (CAT): web service for searching and analyzing carbohydrate-active enzymes in a newly sequenced organism using CAZy database. Glycobiology 20, 1574-84. doi: 10.1093/glycob/cwq106

Park, S., Akira, Y., Kazuhiro, K. (2014). "The family Rhodothermaceae," in The Prokaryotes- Other Major Lineages of Bacteria and the Archaea, eds E. Rosenberg, E. F. DeLong, S. Lory, E. Stackebrandt, and F. Thompson (Berlin Heidelberg: Springer-Verlag), 849-856.

Parks, D. H., Imelfort, M., Skennerton, C. T., Hugenholtz, P., and Tyson, G. W. (2014). CheckM: assessing the quality of microbial genomes recovered from isolates, single cells, and metagenomes. Genome Res. 25, 1043-1055. doi: 10.1101/gr.186072.114

Petitjean, C., Deschamps, P., López-García, P., and Moreira, D. (2015). Rooting the domain Archaea by phylogenomic analysis supports the foundation of the new kingdom Proteoarchaeota. Genome Biol. Evol. 7, 191-204. doi: 10.1093/gbe/evu274

Rees, H. C., Grant, W. D., Jones, B. E., and Heaphy, S. (2004). Diversity of Kenyan soda lake alkaliphiles assessed by molecular methods. Extremophiles 8, 63-71. doi: 10.1007/s00792-003-0361-4

Rice, P., Longden, I., and Bleasby, A. (2000). EMBOSS: the European molecular biology open software suite. Trends Genet. 16, 276-277. doi: 10.1016/S01689525(00)02024-2

Rinke, C., Schwientek, P., Sczyrba, A., Ivanova, N. N., Anderson, I. J., Cheng, J., et al. (2013). Insights into the phylogeny and coding potential of microbial dark matter. Nature 499, 431-437. doi: 10.1038/nature 12352

Rodriguez-Valera, F., Ventosa, A., Juez, G., and Imhoff, J. F. (1985). Variation of environmental features and microbial populations with salt concentrations in a multi-pond saltern. Microbiol. Ecol. 11, 107-115. doi: 10.1007/BF020 10483

Say, R. F., and Fuchs, G. (2010). Fructose 1, 6-bisphosphate aldolase/phosphatase may be an ancestral gluconeogenic enzyme. Nature 464, 1077-1081. doi: 10.1038 /nature08884

Sharon, I., and Banfield, J. F. (2013). Genomes from metagenomics. Science 342, 1057-1058. doi: 10.1126/science. 1247023

Siebers, B., Brinkmann, H., Dörr, C., Tjaden, B., Lilie, H., van der Oost, J., et al. (2001). Archaeal fructose-1, 6-bisphosphate aldolases constitute a new family of archaeal type class I aldolase. J. Biol. Chem. 276, 28710-28718. doi: 10.1074/jbc.M103447200

Siebers, B., and Schönheit, P. (2005). Unusual pathways and enzymes of central carbohydrate metabolism in Archaea. Curr. Opin. Microbiol. 8, 695-705. doi: 10.1016/j.mib.2005.10.014

Simachew, A., Lanzén, A., Gessesse, A., and Øvreås, L., (2015). Prokaryotic community diversity along an increasing salt gradient in a soda ash concentration pond. Microb. Ecol. 71, 326-338. doi: 10.1007/s00248-0150675-7 
Sorokin, D. Y., Abbas, B., Geleijnse, M., Pimenov, G. N., Sukhacheva, M. V., and van Loosdrecht, M. C. (2015b). Methanogenesis at extremely haloalkaline conditions in soda lakes of Kulunda Steppe (Altai, Russia). FEMS Microbiol. Ecol. 91:fiv016. doi: 10.1093/femsec/fiv016

Sorokin, D. Y., Banciu, H. L., and Muyzer, G. (2015a). Functional microbiology of soda lakes. Curr. Opin. Microbiol. 25, 88-96. doi: 10.1016/j.mib.2015. 05.004

Sorokin, D. Y., Berben, T., Melton, E. D., Overmars, L., Vavourakis, C. D., and Muyzer, G. (2014). Microbial diversity and biogeochemical cycling in soda lakes. Extremophiles 18, 791-809. doi: 10.1007/s00792-014-0670-9

Sorokin, D. Y., Janssen, A. J., and Muyzer, G. (2012). Biodegradation potential of halo (alkali) philic prokaryotes. Crit. Rev. Environ. Sci. Technol. 42, 811-856. doi: 10.1080/10643389.2010.534037

Sorokin, D. Y., Kublanov, I. V., Gavrilov, S. N., Rojo, D., Roman, P., Golyshin, P. N., et al. (2015c). Elemental sulfur and acetate can support life of a novel strictly anaerobic haloarchaeon. ISME J. 9, 240-252. doi: 10.1038/ismej.2015.79

Sorokin, D. Y., Toshchakov, S. V., Kolganova, T. V., and Kublanov, I. V. (2015d). Halo(natrono)archaea isolated from hypersaline lakes utilize cellulose and chitin as growth substrates. Front. Microbiol. 6:942. doi: 10.3389/fmicb.2015.00942

Sorokin, D. Y., Tourova, T. P., Galinski, E. A., Belloch, C., and Tindall, B. J. (2006). Extremely halophilic denitrifying bacteria from hypersaline inland lakes Halovibrio denitrificans sp. nov. and Halospina denitrificans gen. nov., sp. nov., and evidence that the genus name Halovibrio (Fendrich 1989) with the type species H. variabilis should be associated with DSM 3050. Int. J. Syst. Evol. Microbiol. 56, 379-388. doi: 10.1099/ijs.0.63964-0

Sorokin, D. Y., Tourova, T. P., Kuznetsov, B. B., Bryantseva, I. A., and Gorlenko, V. M. (2000). Roseinatronobacter thiooxidans gen. nov., sp. nov., a new alkaliphilic aerobic bacteriochlorophyll a-containing bacterium isolated from a soda lake. Microbiology 69, 75-82. doi: 10.1007/BF02757261

Tamura, K., Strecher, G., Peterson, D., Filipski, A., and Kumar, S. (2013). MEGA6: molecular evolutionary genetics analysis version 6. Mol. Biol. Evol. 30, 2725-2729. doi: 10.1093/molbev/mst197

Tatusov, R. L., Fedorova, N. D., Jackson, J. D., Jacobs, A. R., Kiryutin, B., Koonin, E. V., et al. (2003). The COG database: an updated version includes eukaryotes. BMC Bioinformatics 4:41. doi: 10.1186/1471-2105-4-41

Tindall, B. J. (1988). "Prokaryotic life in the alkaline, saline, athalassic environment," in Halophilic Bacteria, ed F. Rodriguez-Valera (Boca Raton, FL: CRC Press), 31-67.

Tourova, T. P., Grechnikova, M. A., Kuznetsov, B. B., and Sorokin, D. Y. (2014). Phylogenetic diversity of bacteria in soda lake stratified sediments. Microbiology 83, 869-879. doi: 10.1134/S0026261714060186

Tourova, T. P., Kovaleva, O. L., Bumazhkin, B. K., Patutina, E. O., Kuznetsov, B. B., Bryantseva, I. A., et al. (2011). Application of ribulose-1, 5-bisphosphate carboxylase/oxygenase genes as molecular markers for assessment of the diversity of autotrophic microbial communities inhabiting the upper sediment horizons of the saline and soda lakes of the Kulunda Steppe. Microbiology 80, 812-825. doi: 10.1134/S0026261711060221

Tremblay, J., Singh, K., Fern, A., Kirton, E. S., He, S., Woyke, T., et al. (2015). Primer and platform effects on $16 \mathrm{~S}$ rRNA tag sequencing. Front. Microbiol. 6:771. doi: 10.3389/fmicb.2015.00771

Tyson, G. W., Chapman, J., Hugenholtz, P., Allen, E. E., Ram, R. J., Richardson, P. M. et al. (2004) Community structure and metabolism through reconstruction of microbial genomes. Nature 428, 37-43. doi: 10.1038/nature02340

Ugalde, J. A., Podell, S., Narasingarao, P., Allen, E. E. (2011). Xenorhodopsins, an enigmatic new class of microbial rhodopsins horizontally transferred between archaea and bacteria. Biol. Direct 6:52. doi: 10.1186/1745-6150-6-52

Urios, L., Agogué, H., Lesongeur, F., Stackebrandt, E., and Lebaron, P. (2006) Balneola vulgaris gen. nov., sp. nov., a member of the phylum Bacteroidetes from the north-western Mediterranean Sea. Int. J. Syst. Evol. Microbiol. 56, 1883-1887. doi: 10.1099/ijs.0.64285-0

Vaisman, N., and Oren, A. (2009). Salisaeta longa gen. nov., sp. nov., a red, halophilic member of the Bacteroidetes. Int. J. Syst. Evol. Microbiol. 59, 2571-2574. doi: 10.1099/ijs.0.010892-0

Wang, Y. X., Li, Y. P., Liu, J. H., Xiao, W., Lai, Y. H., Li, Z. Y., et al. (2013). Gracilimonas mengyeensis sp. nov., a moderately halophilic bacterium isolated from a salt mine in Yunnan, south-western China. Int. J. Syst. Evol. Microbiol. 63, 3989-3993. doi: 10.1099/ijs.0.052043-0

Wood, D., Setubal, J., Kaul, R., Monks, D., Kitajima, J., Okura, V., et al. (2001). The genome of the natural genetic engineer Agrobacterium tumefaciens C58. Science 294, 2317-2323. doi: 10.1126/science.1066804

Yoon, J. H., Kang, S. J., Jung, Y. T., and Oh, T. K. (2009). Psychroflexus salinarum sp. nov., isolated from a marine solar saltern. Int. J. Syst. Evol. Microbiol. 59, 2404-2407. doi: 10.1099/ijs.0.008359-0

Youssef, N. H., Savage-Ashlock, K. N., McCully, A. L., Luedtke, B., Shaw, E. I., Hoff, W. D., et al. (2014). Trehalose/2-sulfotrehalose biosynthesis and glycinebetaine uptake are widely spread mechanisms for osmoadaptation in the Halobacteriales. ISME J. 8, 636-649. doi: 10.1038/ismej.2013.165

Conflict of Interest Statement: The authors declare that the research was conducted in the absence of any commercial or financial relationships that could be construed as a potential conflict of interest.

Copyright (C) 2016 Vavourakis, Ghai, Rodriguez-Valera, Sorokin, Tringe, Hugenholtz and Muyzer. This is an open-access article distributed under the terms of the Creative Commons Attribution License (CC BY). The use, distribution or reproduction in other forums is permitted, provided the original author(s) or licensor are credited and that the original publication in this journal is cited, in accordance with accepted academic practice. No use, distribution or reproduction is permitted which does not comply with these terms. 